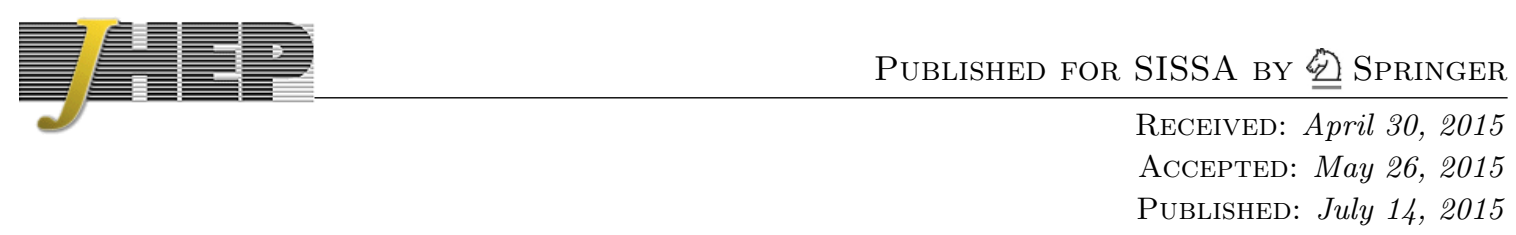

\title{
Uncovering Natural Supersymmetry via the interplay between the LHC and direct Dark Matter detection
}

\author{
Daniele Barducci, ${ }^{a}$ Alexander Belyaev, ${ }^{b, c}$ Aoife K.M. Bharucha, ${ }^{d, e}$ Werner Porod $^{f}$ \\ and Veronica Sanz ${ }^{g}$ \\ ${ }^{a}$ LAPTh, Université Savoie Mont Blanc, CNRS, \\ 9 Chemin de Bellevue, B.P. 110, F-74941 Annecy le-Vieux, France \\ ${ }^{b}$ School of Physics and Astronomy, University of Southampton, \\ Highfield, Southampton SO17 1BJ, U.K. \\ ${ }^{c}$ Particle Physics Department, Rutherford Appleton Laboratory, \\ Chilton, Didcot, Oxon OX11 OQX, U.K. \\ ${ }^{d}$ Physik Department T31, Technische Universität München, \\ James-Franck-Straße 1, D-85748 Garching, Germany \\ ${ }^{e}$ CNRS, Aix Marseille U., U. de Toulon, CPT, UMR 7332, \\ F-13288, Marseille, France \\ ${ }^{f}$ Institut für Theoretische Physik und Astrophysik, Universität Würzburg, \\ D-97074 Würzburg, Germany \\ ${ }^{g}$ Department of Physics and Astronomy, University of Sussex, \\ Brighton BN1 9QH, U.K. \\ E-mail: barducci@lapth.cnrs.fr, a.belyaev@soton.ac.uk, \\ aoife.bharucha@cpt.univ-mrs.fr, porod@physik.uni-wuerzburg.de, \\ v.sanz@sussex.ac.uk
}

ABSTRACT: We have explored Natural Supersymmetry (NSUSY) scenarios with low values of the $\mu$ parameter which are characterised by higgsino-like Dark Matter (DM) and compressed spectra for the lightest MSSM particles, $\chi_{1}^{0}, \chi_{2}^{0}$ and $\chi_{1}^{ \pm}$. This scenario could be probed via monojet signatures, but as the signal-to-background ratio $(\mathrm{S} / \mathrm{B})$ is low we demonstrate that the $8 \mathrm{TeV}$ LHC cannot obtain limits on the DM mass beyond those of LEP2. On the other hand, we have found, for the $13 \mathrm{TeV}$ run of the LHC, that by optimising kinematical cuts we can bring the S/B ratio up to the 5(3)\% level which would allow the exclusion of the DM mass up to 200(250) GeV respectively, significantly extending LEP2 limits. Moreover, we have found that LUX/XENON1T and LHC do play very complementary roles in exploring the parameter space of NSUSY, as the LHC has the capability to access regions where DM is quasi-degenerate with other higgsinos, which are challenging for direct detection experiments.

Keywords: Supersymmetry Phenomenology, Monte Carlo Simulations ARXIV EPRINT: 1504.02472 


\section{Contents}

1 Introduction 1

2 Parameter space and spectrum of NSUSY 3

3 Dark Matter direct and indirect detection in the NSUSY parameter $\begin{array}{ll}\text { space } & 7\end{array}$

4 LHC potential to probe NSUSY 10

4.1 Analysis setup 11

4.2 LHC Run1: the reach of monojet searches 13

$\begin{array}{lll}4.3 & 13 \mathrm{TeV} \text { LHC potential and complementarity to underground experiments } & 14\end{array}$

5 Conclusions

\section{Introduction}

The naturalness of Supersymmetry (SUSY), which has been subjected to much discussion and thorough investigation for more than two decades [1-57], has become even more relevant now, as the Large Hadron Collider (LHC) collaborations ATLAS and CMS have started to probe SUSY in the TeV region. Indeed, the lack of evidence for superparticles at the CERN LHC, along with the rather high value of the Higgs boson mass in the context of the Minimal Supersymmetric Standard Model (MSSM), raises the question of whether the remaining allowed parameter space suffers from a high degree of fine-tuning, and if there is any parameter space of Natural SUSY (NSUSY) left. We discuss this problem in the framework of the well motivated MSSM.

Based on standard measures of fine tuning [1, 2], the NSUSY parameter space was originally associated with light higgsinos, gluinos and stops, the SUSY partners of the SM Higgs, gluons and top quark. The present LHC limits on the masses of the latter two are approaching the $\mathrm{TeV}$ scale, under the assumption that the mass gap between these states and the lightest supersymmetric particle (LSP) , $\tilde{\chi}_{1}^{0}$, is large enough (see for example [58$62])$. Here $\tilde{\chi}_{1}^{0}$ is the lightest of the four neutralinos, the mass eigenstates arising from the mixing of the fermionic component of the Higgs and gauge superfields, $\left(\tilde{H}_{d}^{0}, \tilde{H}_{u}^{0}\right)$ and $\left(\tilde{B}^{0}, \tilde{W}^{0}\right)$, which are commonly known as the higgsinos, the bino and the wino. Moreover, these limits have a certain degree of model dependence and for stops, $\tilde{t}_{1,2}$, the experimental limits rely on certain decay channels being dominant (e.g. $\tilde{t}_{1} \rightarrow t \tilde{\chi}_{1}^{0}$, with $t$ the top-quark) or on a substantial mass splitting between $\tilde{t}_{1}$ and $\tilde{\chi}_{1}^{0}$, and can be significantly relaxed. For example, in the scenario under consideration in this paper with higgsino like dark matter (DM), the stop branching ratios strongly depend on the left-right admixture of the lightest stop. Therefore model independent collider bounds on stops are weak or non-existent. 
It has however been shown that usual fine-tuning measures, defined as the sensitivity of the weak scale to fractional variations in the fundamental parameters of the theory, can be low even if the masses of the supersymmetric scalars are large. This happens in the so called "hyperbolic branch"(HB) [6] or "focus point" (FP) [14, 15, 63] regions of the minimal super gravity (mSUGRA) parameter space, where the value of the Higgs mass parameter, $\mu$, can be low if the universal gaugino mass $M_{1 / 2}$ is not too large, as a consequence of the subtle interplay between the electroweak (EW) gauge couplings and the top-Yukawa coupling in the evolution of the squared Higgs mass parameters using the renormalization group equations (RGE). Moreover it was recently argued [64] that EW fine-tuning in SUSY scenarios can be grossly overestimated by neglecting additional terms, stemming from the ultra-violet (UV) completion of the model, that can lead to large cancellations favouring a low $\mu$ parameter, but not necessarily a low stop mass up to a certain limit. Taking this point of view, we will take a low $\mu$ parameter to be the definition of NSUSY throughout our study.

In the case $\mu \ll M_{1}, M_{2}$ (the EW gaugino mass parameters) one finds that the three lightest neutralino and chargino mass eigenstates, $\tilde{\chi}_{1}^{0}, \tilde{\chi}_{2}^{0}$ and $\tilde{\chi}_{1}^{ \pm}$, are quasi-degenerate and that these states are nearly pure higgsinos. In this scenario the DM relic density is typically below the WMAP [65] and PLANCK [66] measurements, because of the high rate of higgsino annihilation to standard model (SM) gauge and Higgs bosons and the higgsino coannihilation processes $[67,68]$. One should note that this parameter space with the relic abundance below the experimental constraints is not however excluded, since the remaining relic abundance can be accounted for by other additional sources, e.g. axions. This NSUSY scenario, which is characterised by relatively light higgsinos in comparison to other SUSY particles, is not just motivated by its simplicity, but also by the lack of evidence for SUSY to date. We take advantage of the fact that NSUSY scenarios can be effectively described by a two dimensional parameter space, defined by the DM mass, i.e. the mass of $\tilde{\chi}_{1}^{0}$, and $\Delta \mathrm{M}$, the mass difference between the DM candidate and the next to lightest supersymmetric (NLSP), typically $\tilde{\chi}_{1}^{ \pm}$, and our study explores the complementarity of the LHC and direct detection (DD) DM search experiments in covering this region. Such complementarity was the subject of recent studies, see e.g. [69] and references there in.

It was already shown a decade ago that the $\mathrm{HB} / \mathrm{FP}$ parameter space is challenging to probe at the LHC [70] even if the mass gap between $\tilde{\chi}_{1,2}^{0}$ and $\tilde{\chi}_{1}^{ \pm}$is large enough to provide leptonic signatures. The most challenging case arises when the mass gap between these states is too small to produce any detectable leptons. The only way to probe such a scenario is via mono-object signatures, i.e. signatures involving a high transverse momentum particle recoiling against missing transverse emerge $\left(E_{T}^{\text {miss }}\right)$, of which the monojet signature is particularly of relevance at the LHC, as initially suggested in [71] for generic compressed spectra.

This technique has already been used in studies of quasi-degenerate higgsino spectra via monojet $+E_{T}^{\text {miss }}$ and monojet $+E_{T}^{\text {miss }}+$ soft di-lepton signatures for the NSUSY parameter space we consider [72-76]. However, we believe that these analyses are not entirely complete and/or have certain drawbacks. For example, in [72] the 95\% confidence level (CL) reach for the $14 \mathrm{TeV}$ run of the LHC was calculated assuming a signal (S) to background (B) ratio 
below the $2 \%$ level, which is probably not quite feasible when taking into account that the actual systematic error should be above $3-5 \%$ even for quite optimistic analyses [77-79]. In [74] the authors have performed their analysis at the parton level while, as shown in a preliminary analysis $[80,112]$, a fast detector simulation analysis leads to qualitatively different results and, therefore, is crucial. In [75] the conclusion about the observability of the quasi-degenerate higgsino NSUSY scenario from the monojet search was negative. However in this study the authors did not attempt to optimise the $E_{T}^{\text {miss }}$ which turns out to be important as we will show in this paper. In [73] the prospects were more optimistic even after including systematics uncertainties. However, also there no optimisation of the cuts was performed which considerably enhances the accessible mass range as we will show. One should also note the Ref. [76] where authors suggested a new promising signature including a pair of soft leptons, and have demonstrated its potential power. However, in this study the important $b \bar{b}$ background was not considered as we discuss below, implying thus further background investigation for this signature which we do not consider at present. Finally one should mention Ref. [81], which studied similar to [76] monojet plus soft lepton signature suggesting visibly harder cuts to suppress $b \bar{b}$ background. The respective higgsino mass reach from this study is quite limited. On the other hand the suggested b-jet veto will not quite work for the signature with isolated soft muons, so, we believe that one should estimate $b \bar{b}$ background more precisely even for the case of harder cuts suggested in [81].

Motivated by the above-mentioned previous studies, here we aim to perform a comprehensive and realistic analysis of the monojet potential to probe this NSUSY scenario. Our analysis is performed at the level of a fast detector simulation and the whole twodimensional NSUSY parameter space mentioned above, rather than selected benchmarks as were attempted previously, is explored therefore completely covering the region of our interest. We consider prudent systematic errors and optimise the kinematic cuts to keep the $\mathrm{S} / \mathrm{B}$ ratio at a reasonable level. We then discuss the LHC potential to cover the NSUSY parameter space at $8 \mathrm{TeV}$ and produced projections for the $13 \mathrm{TeV}$ run of the CERN machine. By analysing also the exclusion potentiality for DM direct detection experiments, we aim to show that collider and DD experiments have a high degree of complementarity.

The paper is organised as follows. In section 2 we describe the parameter space and mass spectrum of NSUSY while in section 3 we discuss the DM properties of this scenario. section 4 is dedicated to the analysis of the collider phenomenology of the compressed higgsino scenario while in section 5 we show the complementarity of collider and the DD experiment. We conclude in section 6 .

\section{Parameter space and spectrum of NSUSY}

In the bases $\left(\tilde{B}^{0}, \tilde{W}^{0}, \tilde{H}_{d}^{0}, \tilde{H}_{u}^{0}\right)$ and $\left(\tilde{W}^{0}, \tilde{H}_{d}^{0}\right)$ the mass matrices of the neutralino and chargino sector of the MSSM are

$$
M_{\tilde{\chi}_{0}^{0}}=\left(\begin{array}{cccc}
M_{1} & 0 & -M_{Z} s_{\omega} c_{\beta} & M_{Z} s_{\omega} s_{\beta} \\
0 & M_{2} & M_{Z} c_{\omega} c_{\beta} & -M_{Z} c_{\omega} s_{\beta} \\
-M_{Z} s_{\omega} c_{\beta} & M_{Z} c_{\omega} c_{\beta} & & -\mu \\
M_{Z} s_{\omega} s_{\beta} & -M_{Z} c_{\omega} s_{\beta} & -\mu & 0
\end{array}\right) \quad M_{\tilde{\chi}_{1}^{ \pm}}=\left(\begin{array}{cc}
M_{2} & \sqrt{2} M_{W} s_{\beta} \\
\sqrt{2} M_{W} c_{\beta} & \mu
\end{array}\right)
$$


where $M_{1}$ and $M_{2}$ are the soft susy breaking mass parameter for $\tilde{B}$ and $\tilde{W}, \mu$ is the Higgsino mass parameter, $c_{\omega}$ and $s_{\omega}$ are $\cos$ and $\sin$ of the Weinberg angle, $\tan \beta=v_{2} / v_{1}$ is the ratio of the vacuum expectation values of two Higgs doublets, $s_{\beta}, c_{\beta}$ are $\sin \beta$ and $\cos \beta$, respectively and $m_{Z}, m_{W}$ are the masses of the SM gauge bosons $Z^{0}$ and $W^{ \pm}$

As a first step we consider scenarios where the $\tilde{\chi}_{1}^{0}$ and $\tilde{\chi}_{1}^{ \pm}$have a high Higgsino component with all the SUSY partners of the SM fermions with masses in the multi $\mathrm{TeV}$ range and, to first obtain a qualitative understanding of the spectrum, we then expand the corresponding mass eigenvalues in the limit $|\mu| \ll\left|M_{1}\right|,\left|M_{2}\right|$ obtaining:

$$
\begin{aligned}
& m_{\tilde{\chi}_{1,2}^{0}} \simeq \mp\left[|\mu| \mp \frac{m_{Z}^{2}}{2}\left(1 \pm s_{2 \beta}\right)\left(\frac{s_{\omega}^{2}}{M_{1}}+\frac{c_{\omega}^{2}}{M_{2}}\right)\right] \\
& m_{\tilde{\chi}_{1}^{ \pm}} \simeq|\mu|\left(1+\frac{\alpha\left(m_{Z}\right)}{\pi}\left(2+\ln \frac{m_{Z}^{2}}{\mu^{2}}\right)\right)-s_{2 \beta} \frac{m_{W}^{2}}{M_{2}}
\end{aligned}
$$

where we have defined $s_{2 \beta}=\sin (2 \beta) \operatorname{sgn}(\mu)$ and $\alpha$ is the electromagnetic structure constant. In the case of $\tilde{\chi}_{1}^{ \pm}$we have also included the electromagnetic corrections as this shifts the mass by about $0.5 \%$ which is indeed important in those cases where the mass splitting between $\tilde{\chi}_{1}^{ \pm} / \tilde{\chi}_{2}^{0}$ and $\tilde{\chi}_{1}^{0}$ is of the order of a few $\mathrm{GeV}$. We would like to note, that in our numerical results we have included the complete one-loop corrections in the calculation of the masses as this changes the absolute mass scale by several per-cent. However, the mass splittings discussed below are hardly affected as the additional corrections changes those at most by $O\left(\alpha /(4 \pi) \ln \left(m_{W}^{2} / m_{Z}^{2}\right)\right.$ which is below the per-mile level. For positive (negative) $\mu$ the mass eigenstate with negative (positive) CP-eigenvalue is the lightest one. The mass splittings are given by

$$
\begin{aligned}
\Delta m_{0} & =m_{\tilde{\chi}_{2}^{0}}-m_{\tilde{\chi}_{1}^{0}} \simeq m_{Z}^{2}\left(\frac{s_{\omega}^{2}}{M_{1}}+\frac{c_{\omega}^{2}}{M_{2}}\right) \\
\Delta m_{ \pm} & =m_{\tilde{\chi}_{1}^{ \pm}}-m_{\tilde{\chi}_{1}^{0}} \simeq \frac{\Delta m_{0}}{2}+|\mu| \frac{\alpha\left(m_{Z}\right)}{\pi}\left(2+\ln \frac{m_{Z}^{2}}{\mu^{2}}\right)
\end{aligned}
$$

where we have neglected corrections of the order $1 / \tan \beta$ and $\left(\mu / M_{1,2}\right)^{2}$.

In order to analyse scenarios where the mass splitting between $\tilde{\chi}_{1}^{ \pm}$and $\tilde{\chi}_{1}^{0}$ varies from the (quasi) degenerate regime up to the regime with larger mass splittings, we have chosen the following range of parameter space:

$\mu=(100,300) \mathrm{GeV} \quad M_{1}=(\mu, \mu+600) \mathrm{GeV}$ and $(-\mu,-\mu-600) \mathrm{GeV} \tan \beta=(5-50)$,

fixing the value of $M_{2}=2 \mathrm{TeV}$, which has the effect of decoupling $\chi_{4}^{0}$ and $\chi_{2}^{ \pm}$that will not be considered anymore in the following, along with the rest of SUSY spectrum, which is assumed to be decoupled.

For $\left|M_{1}\right| \simeq \mu$ one can obtain a simple approximation for $\tilde{\chi}_{1,2,3}^{0}$ masses [82] which we confirm by numerical evaluation, indicating that in this parameter region $\tilde{\chi}_{1}^{0}$ and $\tilde{\chi}_{3}^{0}$ are strongly mixed bino-higgsino states whereas $\tilde{\chi}_{2}^{0}$ is essentially a higgsino-like state with only a small bino component.

The mass splitting $\Delta \mathrm{M}=m_{\tilde{\chi}_{1}^{ \pm}}-m_{\tilde{\chi}_{1}^{0}}$ is shown in figure 1 as a function of $\mu$ and $M_{1}$ for the case of positive and negative $M_{1}$ while in figure 2 we show the contour lines for 

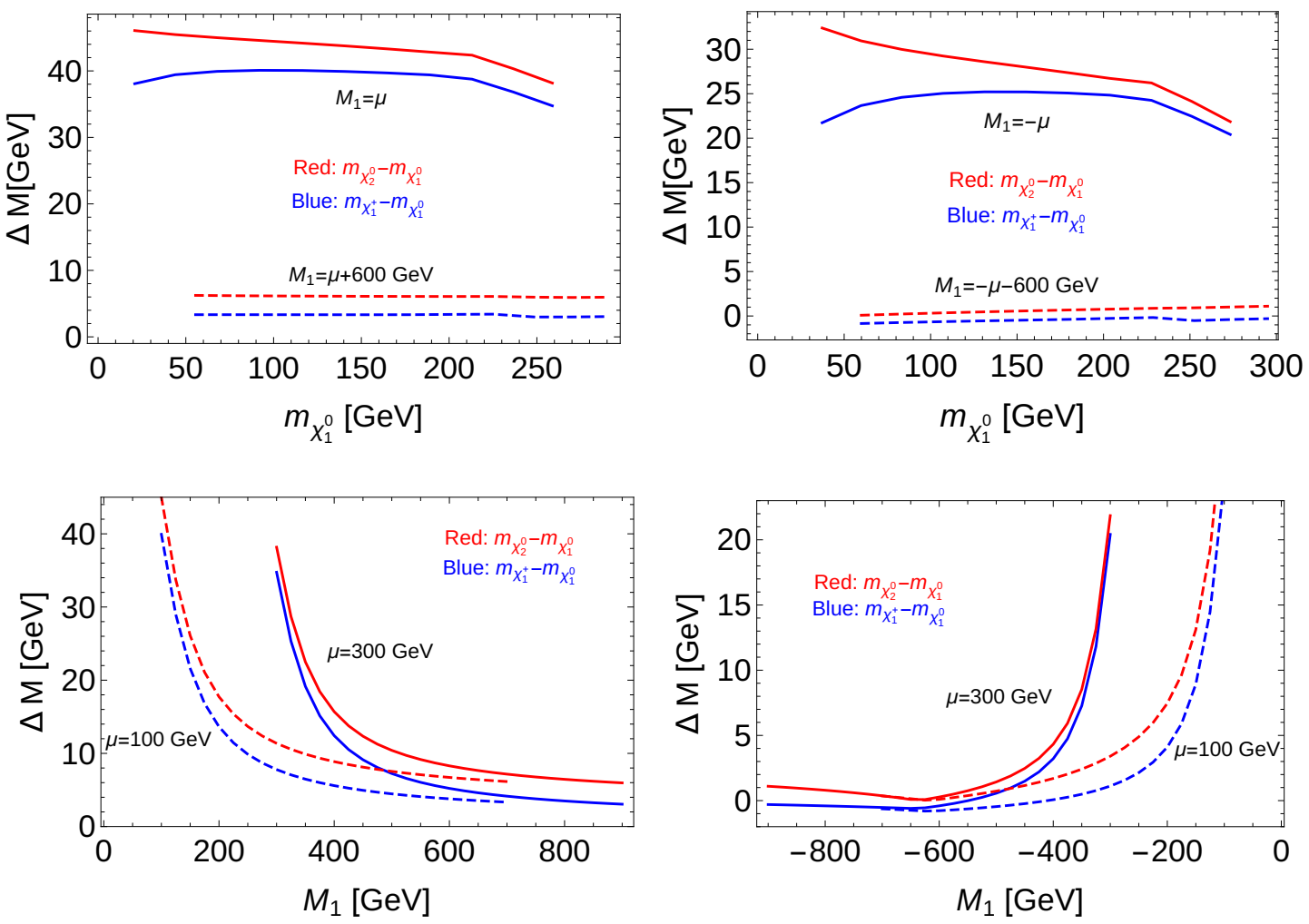

Figure 1. $\tilde{\chi}_{1}^{ \pm}-\tilde{\chi}_{1}^{0}$ and $\tilde{\chi}_{2}^{0}-\tilde{\chi}_{1}^{0}$ mass splitting values as a function of $m_{\chi_{1}^{0}}$ (upper row) and $M_{1}$ (lower row) for the case $M_{1}>0$ (left) and $M_{1}<0$ (right).

$\tilde{\chi}_{1}^{ \pm}-\tilde{\chi}_{1}^{0}$ mass splitting. The relation between $\left|M_{1}\right|-\mu$ and the value of the mass splitting, which runs from quasi-degenerate scenario, $\Delta \mathrm{M} \simeq 1-5 \mathrm{GeV}$ for large $M_{1}$, to bigger values, $\Delta \mathrm{M} \simeq 10-30 \mathrm{GeV}$ for $\left|M_{1}\right| \simeq \mu$, is clearly shown in this plots where the mass of the lightest neutralino, the DM candidate, is also presented.

The nature of the neutralinos and charginos, as well as the small mass splitting between them, has a strong impact on their decay modes. In the case of pure higgsinos, the three body decays are dominated by virtual vector bosons, and due to the small mass differences the decays into third generation fermions are suppressed. Note that in the scenario where $M_{1}$ is close to $|\mu|$, the off-shell lightest Higgs boson, $h^{0}$, can also give sizeable contributions $[83,84]$. Also, one should note that in the case $\left|M_{1}\right| \simeq \mu, m_{\tilde{\chi}_{3}^{0}}-m_{\tilde{\chi}_{1}^{0}} \simeq 2\left(m_{\tilde{\chi}_{2}^{0}}-m_{\tilde{\chi}_{1}^{0}}\right)$ and $\tilde{\chi}_{3}^{0}$ decays to the lightest chargino with the $50 \%$ probability while sharing about $25 \%$ decay to each of $\tilde{\chi}_{1}^{0}$ and $\tilde{\chi}_{2}^{0}$.

Three body decays in the limit of small mass separation are discussed in [85], where an effective theory study of the pseudo-Dirac DM scenario [86-88] such as the higgsino-like was performed. In this limit the decay width does not depend on the overall neutralino mass, but just on the mass difference

$$
\Gamma\left(\tilde{\chi}_{1}^{ \pm}, \tilde{\chi}_{2}^{0} \rightarrow f f^{\prime} \tilde{\chi}_{1}^{0}\right)=\frac{C^{4}}{128 \pi^{3}} \frac{\Delta m^{5}}{\Lambda^{4}}
$$

where $\Lambda \simeq m_{W, Z, h^{0}}$ is the mass of the leading mediator, while $\Delta m$ is either $m_{\tilde{\chi}_{2}^{0}}-m_{\tilde{\chi}_{1}^{0}}$ 

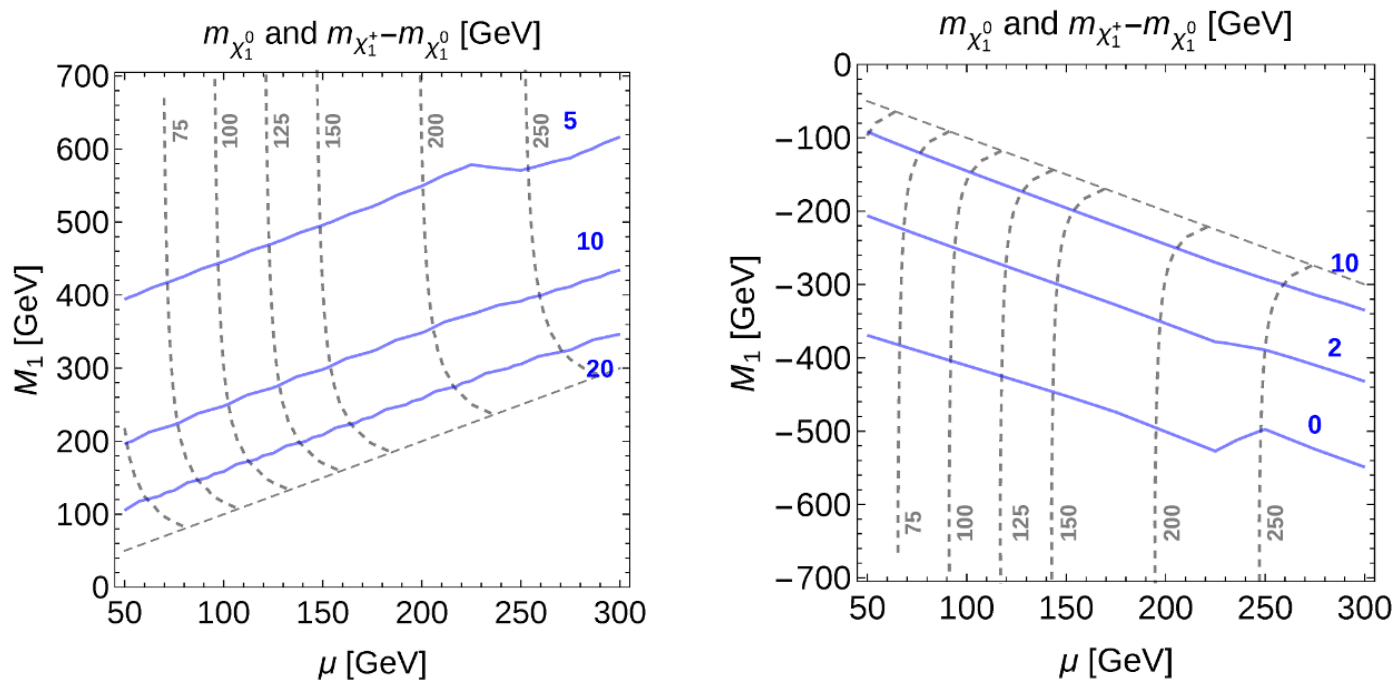

Figure 2. Mass splitting between $\tilde{\chi}_{1}^{ \pm}$and $\tilde{\chi}_{1}^{0}$ (blue solid line) and mass of $\tilde{\chi}_{1}^{0}$ (gray dashed line) in the $\mu$ - $M_{1}$ plane.

or $m_{\tilde{\chi}_{1}^{ \pm}}-m_{\tilde{\chi}_{1}^{0}}$. As an example for off-shell $Z$ exchange and decay into leptons, the coefficient $C$ is

$$
C^{4} \simeq \frac{1}{4} \frac{g^{4}}{c_{\omega}^{4}}\left(\left(s_{\omega}^{2}-1 / 2\right)^{2}+s_{\omega}^{4}\right)
$$

and a similar expression occurs for the case of the off-shell $W$-decay. The proper decay length is very sensitive to the value of $\Delta m$, and values below the GeV lead to displaced vertices, or collider-stable $\tilde{\chi}_{1}^{ \pm}$and $\tilde{\chi}_{2}^{0}$. Indeed, for the decay $\tilde{\chi}_{2}^{0} \rightarrow f \bar{f} \tilde{\chi}_{1}^{0}$ with an off shell $Z$ exchange, the proper decay length is given by

$$
L=c \tau \simeq 0.025 \mathrm{~cm}\left(\frac{\Delta m}{1 \mathrm{GeV}}\right)^{-5}
$$

which implies that for $\Delta m \lesssim 0.1 \mathrm{GeV}, \tilde{\chi}_{2}^{0}$ would be collider stable. Similarly, for $\Delta m \lesssim$ $1 \mathrm{GeV}$ one could look for displaced vertices of order $100 \mu \mathrm{m}$. Note that the measured decay length would depend on the boost factor of the decaying neutralino to be taken into account as discussed in details in ref. [85]. While collider stable $\tilde{\chi}_{2}^{0}$ will contribute to the $E_{T}^{\text {miss }}$, long lived or collider stable $\tilde{\chi}_{1}^{ \pm}$will provide a clear signature in the detector. A $\tilde{\chi}_{1}^{ \pm}$ with a long enough lifetime can be detected in the tracking detectors by identifying decays that result in tracks with no associated hits in the outer region of the tracking system as recently analysed by ATLAS [89] and CM [90] collaborations. Both collaborations have obtained similar results, concluding on sensitivity for charginos with a lifetime between 0.1 ns and 100 ns and covering chargino mass up to $500 \mathrm{GeV}$ which significantly surpass the reach of the LEP experiments. For $\Delta \mathrm{M} \lesssim 0.25 \mathrm{GeV}$ the chargino could be a collider-stable charged particle [91], and bounds on such a situation arising from the $8 \mathrm{TeV}$ run of the LHC can be estimated to be $m_{\tilde{\chi}^{ \pm}} \gtrsim 300 \mathrm{GeV}$ [92]. On the other hand, we have found that for $\Delta \mathrm{M} \gtrsim 0.4 \mathrm{GeV}$ there is no limit on $m_{\tilde{\chi}_{1}^{ \pm}}>100 \mathrm{GeV}$ from the above LHC searches. 

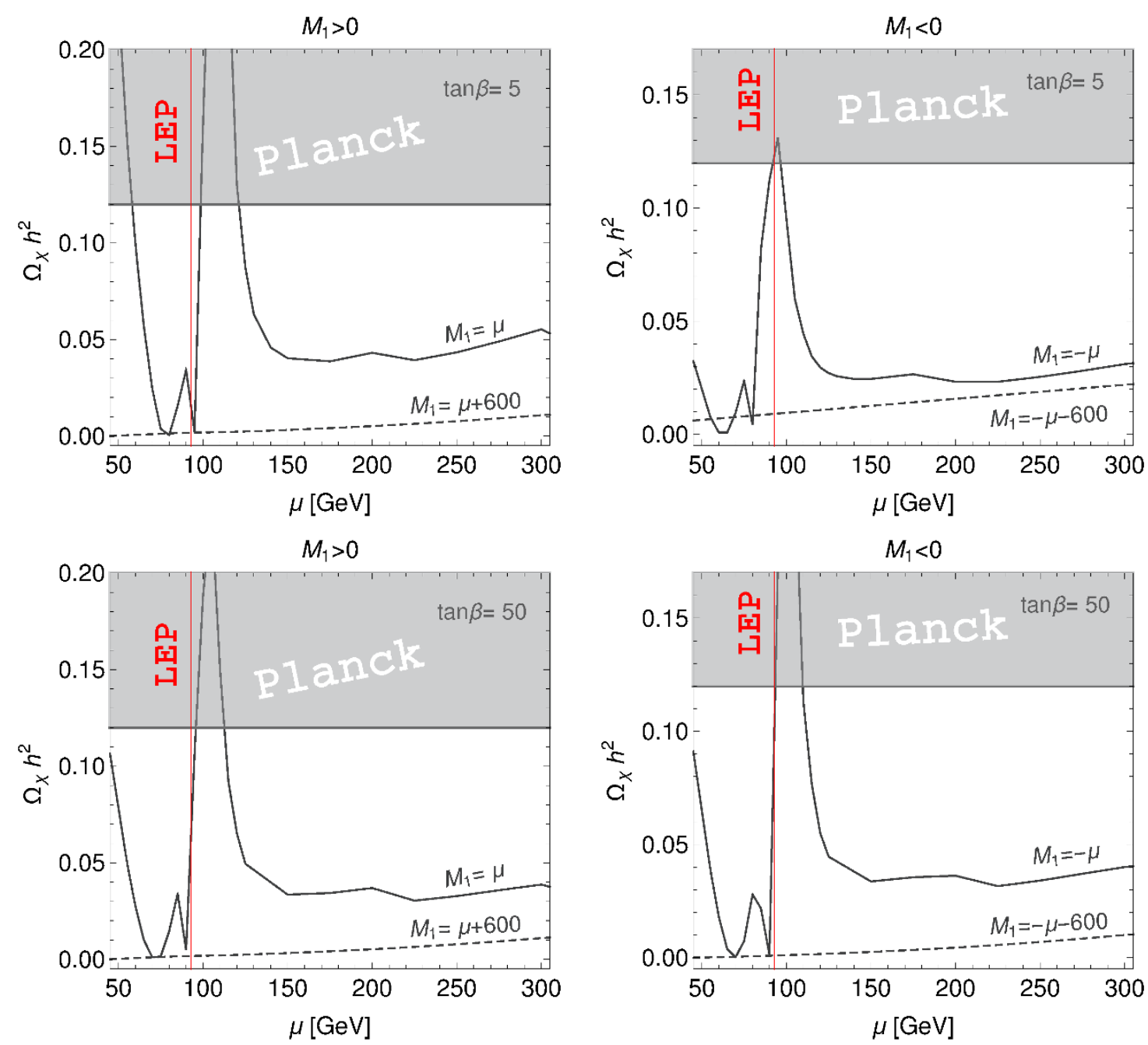

Figure 3. The predicted value of the dark matter relic density $\Omega_{\mathrm{DM}} h^{2}$ is shown as a function of $\mu$ for $\tan \beta=5,50$ and positive (left) or negative (right) values of $M_{1}$ as indicated. The relic density measured by the Planck satellite, $\Omega_{\mathrm{DM}}^{\mathrm{Planck}} h^{2}$, is also shown for comparison, and the region excluded due to an overabundance of DM is indicated in grey.

Therefore, our task is to analyse the potential of the monojet search to cover the NSUSY parameter space with $\Delta \mathrm{M} \gtrsim 0.4 \mathrm{GeV}$.

\section{Dark Matter direct and indirect detection in the NSUSY parameter space}

The results from Planck [66, 93] (see also WMAP [65]) have further decreased the error on the already very precise measurement of the dark matter relic density, $\Omega_{\mathrm{DM}}^{\text {Planck }} h^{2}=$ $0.1184 \pm 0.0012$.

As we assume R-parity to hold, the LSP will be stable and will contribute to this relic density. In the scenarios under consideration, the LSP is the lightest neutralino, $\tilde{\chi}_{1}^{0}$, which is dominantly higgsino-like with a variable bino component. It is well known that for $\tilde{\chi}_{1}^{0}$ of 
mass $\mathcal{O}(100 \mathrm{GeV})$, a higgsino-like LSP provides DM relic density below the level observed by Planck. This happens because of the annihilation and co-annihilation rate of LSP and NLSP particles in the early Universe begin too large. On the other hand, for bino-like neutralinos the annihilation is suppressed, resulting in DM over-abundance. In the mass range we study, mixed bino-higgsino LSPs can therefore lead to the correct relic abundance. For $\mu \lesssim M_{1}$ the LSP is mainly higgsino-like and the value of $\Omega_{\mathrm{DM}} h^{2} \lesssim \Omega_{\mathrm{DM}}^{\text {Planck }} h^{2}$, however this nonetheless at least solves the typical problem of the over-closure of the universe for neutralinos of this mass range in generic SUSY parameter space. In this case we then assume that the remaining relic abundance is accounted for by other means, for example, it could come from multi-TeV moduli field where the higgsino LSP is non-thermally produced (see e.g. ref. [94]) or from mixed axion-higgsino DM (e.g. ref. [30]).

In order to assess the compatibility of the scenarios under our investigation with existing experimental limits, we have evaluated $\Omega_{\mathrm{DM}} h^{2}$, the spin-independent annihilation cross section $\left(\sigma_{\mathrm{SI}}\right)$ and the respective DD rates using micrOMEGAs $2.4 .1[95,96]$. In figure 3 we show the results for $\Omega_{\mathrm{DM}} h^{2}$ as a function of $\mu$ for $\tan \beta=5,50$ and positive or negative values of $M_{1}$. Note that the uncertainty on $\Omega_{\mathrm{DM}} h^{2}$ is not shown, the full one-loop corrections are not yet available, but we expect that these are not too large and will not qualitatively change our conclusions. From these plots we see, as expected, that in general $\Omega_{\mathrm{DM}} h^{2}$ lies below $\Omega_{\mathrm{DM}}^{\text {Planck }} h^{2}$, and decreases as $\tilde{\chi}_{1}^{0}$ becomes increasingly higgsino-like. This is because of the mass splitting between $\tilde{\chi}_{1}^{ \pm}$and $\tilde{\chi}_{1}^{0}$, which becomes larger as $M_{1}$ decreases. This suppresses the coannihilation channels which otherwise lead to an efficient reduction of the relic density. Below the $W W$ threshold, the annihilation of the mixed gaugino-higgsino neutralinos therefore occurs via the Higgs and Z-bosons. The small bottom Yukawa coupling and the suppressed coupling to the $Z$ implies that this mechanism is not efficient, apart from at the $Z$ and $h$ resonance. Therefore the spike in the relic density $\mu \sim 100 \mathrm{GeV}$ can be explained by the fact that this is just below the $W W$ threshold. At lower values of $\mu \sim 70,90 \mathrm{GeV}$ one moreover observes two dips corresponding to the Z-boson and Higgs funnels. This is most pronounced for positive values of $M_{1}$ and lower values of $\tan \beta$ where the mass splitting is larger.

In figure 4 we further show the spin-independent annihilation cross section for DD, again for positive and negative $M_{1}$ as in figure 3 , where instead of $\sigma_{\mathrm{SI}}$ we plot the rescaled quantity $R_{\Omega} \sigma_{\mathrm{SI}}(\mathrm{pb})$, where the scaling factor $R_{\Omega}=\Omega_{\mathrm{DM}} / \Omega_{\mathrm{DM}}^{\text {Planck }}$ allows easy comparison with the most recent limits (also reported in these plots) from LUX [97], as well as the projected limits from XENON1T after 2 years live-time and 1 ton fiducial mass (see e.g. ref. [98]), which in general assume the relic density to be the value measured by Planck. Figure 4 illustrates that the region with a low LSP masses and a higher mass splitting between $\tilde{\chi}_{1}^{ \pm}$and $\tilde{\chi}_{1}^{0}$ (see figure 2), which is also easiest to see at colliders, is in fact excluded as the $\sigma_{\mathrm{SI}}$ for the DD experiments is too high. In the following we will further highlight the interesting complementarity between the reach of the collider searches and the DD searches, particularly interesting for low DM masses. In figure 5 we also show the mass splitting between $\tilde{\chi}_{1}^{ \pm}$and $\tilde{\chi}_{1}^{0}$ in the $\mu-M_{1}$ plane of figure 2 , along with the region excluded by LUX and the projected exlusion regions from XENON1T. This emphasises that the region where $\mu$ and $M_{1}$ are very close each other is already excluded by LUX, which in turns puts an upper bound on the splitting between $\tilde{\chi}_{1}^{ \pm}$or $\tilde{\chi}_{2}^{0}$ and $\tilde{\chi}_{1}^{0}$. Note that 

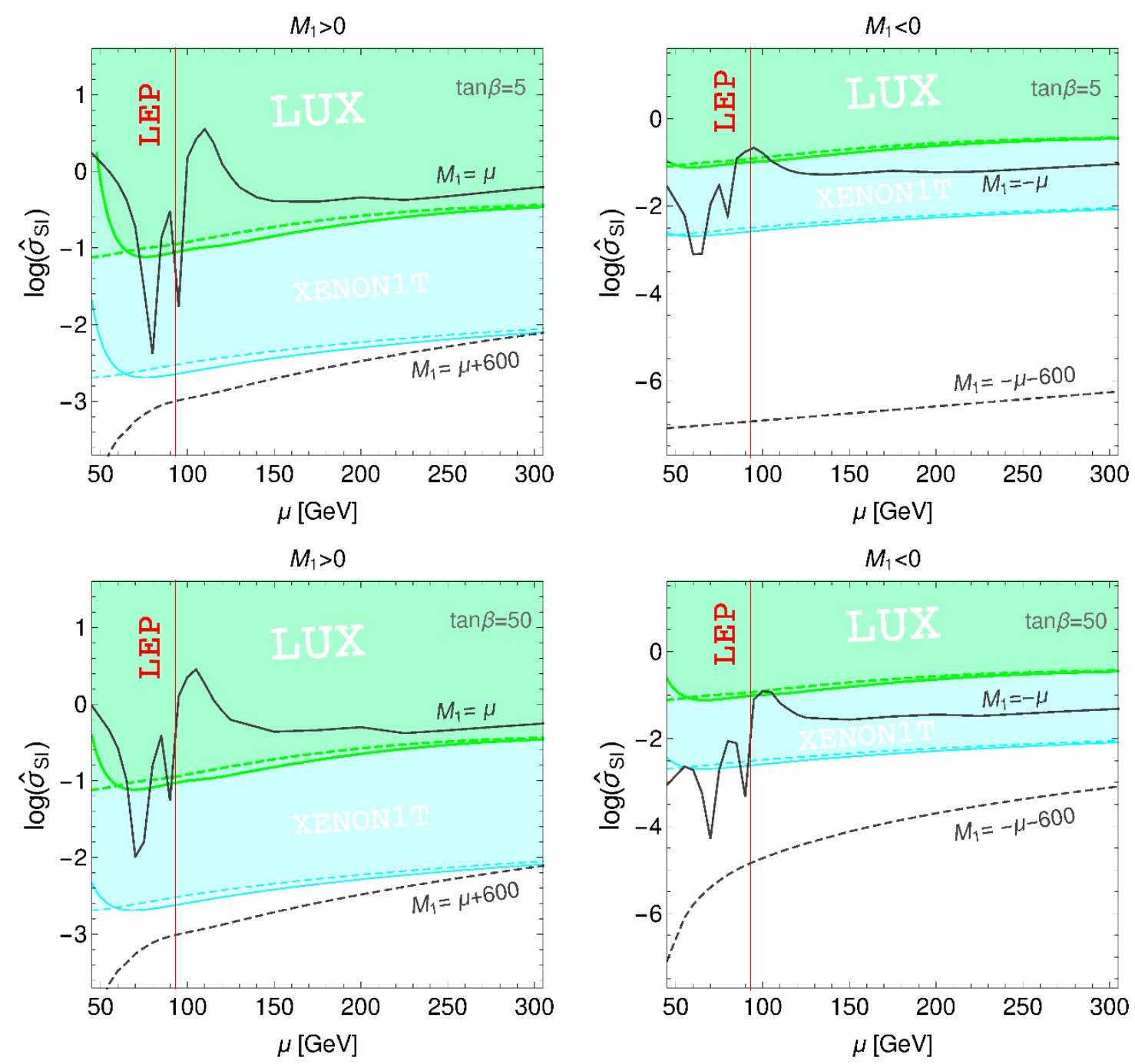

Figure 4. The logarithm of the predicted value of the spin-independent annihilation cross section for $\mathrm{DD} \hat{\sigma}_{\mathrm{SI}}=R_{\Omega} \sigma_{\mathrm{SI}} /\left(10^{-8} \mathrm{pb}\right)$, rescaled by $R_{\Omega} /\left(10^{-8} \mathrm{pb}\right)$ where $R_{\Omega}=\Omega_{\mathrm{DM}} / \Omega_{\mathrm{DM}}^{\text {Planck }}$, is shown as a function of $\mu$ for $\tan \beta=5,50$ and positive (left) or negative (right) values of $M_{1}$ as indicated. The excluded limit from LUX (green), as well as the projected exclusion from XENON1T (cyan) are also shown for comparison, where the solid and dashed lines represent the exclusions for $\left|M_{1}\right|=\mu$ and $\left|M_{1}\right|=\mu+600 \mathrm{GeV}$ respectively.

for positive values of $M_{1}$, the splitting is larger, as is the mixing between bino and higgsino component of the DM. As larger mixing leads to larger couplings to Higgs bosons, DD is more sensitive to the case $M_{1}>0$.

A related question is whether these scenarios could be excluded by indirect detection (ID) experiments, i.e. the detection of energetic $e^{ \pm}, \gamma, p$ or $\bar{p}$, which may be created by the pair annihilation of weakly interacting massive particles (WIMPS). It turns out that the strongest bounds on neutralinos coming from such experiments are set by gamma ray telescopes: both the Fermi-LAT gamma-ray space telescope [99] as well as ground based telescopes. Fermi-LAT is sensitive to gamma rays particularly in the low mass range up to 

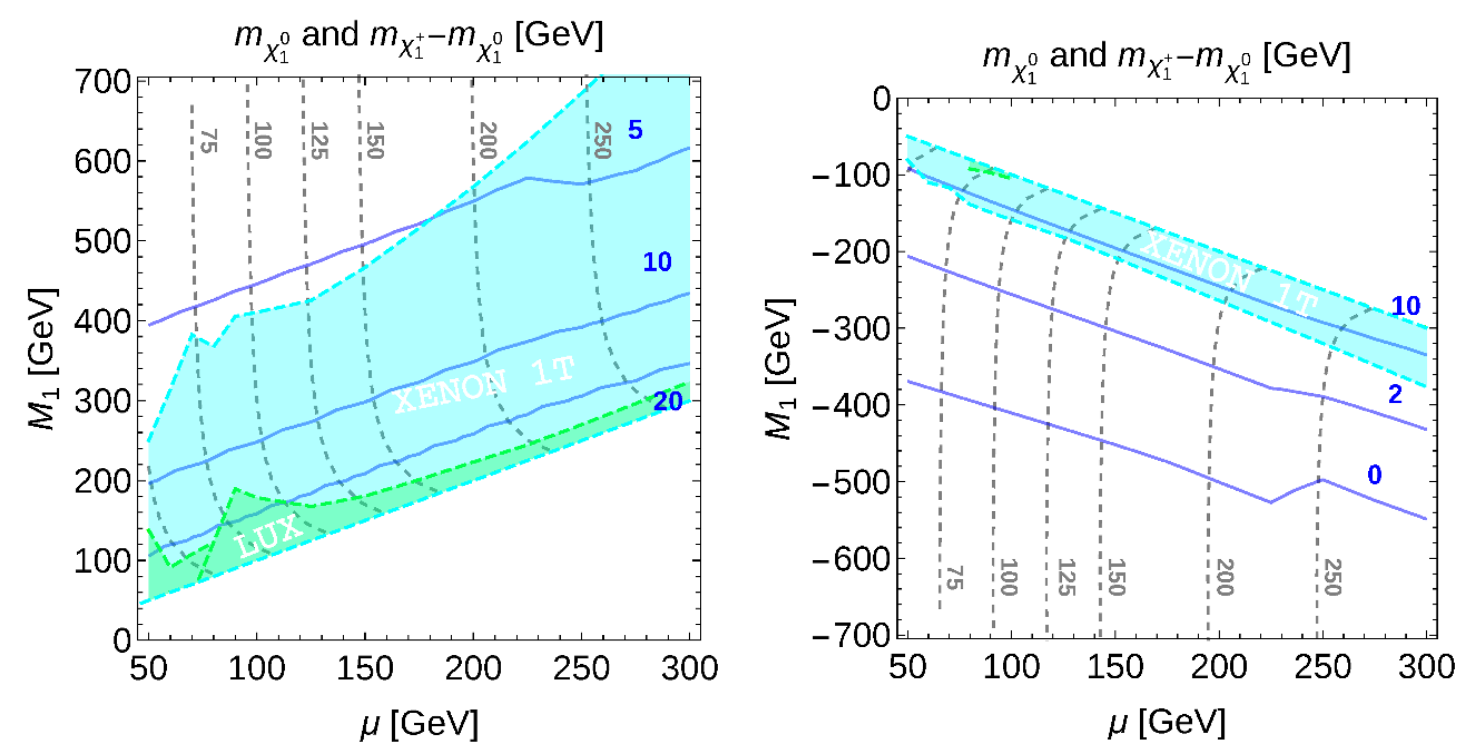

Figure 5. The mass splitting between $\tilde{\chi}_{1}^{ \pm} / \tilde{\chi}_{1}^{0}$ (blue solid line) and mass of $\tilde{\chi}_{1}^{0}$ (black dashed line) is shown in the $\mu-M_{1}$ plane, along with the region excluded by LUX results (green).

$\mathcal{O}(100 \mathrm{GeV})$. It is therefore particularly sensitive to lighter mixed bino-higgsino neutralinos, but the bounds are not competitive with those coming from DD. Here we are interested in light higgsinos with possibly some bino component, for which the relic density is in general much below the value measured at Planck/WMAP (see figure 3). In this case, all bounds from ID must be scaled by the square of the ratio of the predicted relic density to the experimental value. The rescaling appears as the pair annihilation cross section depends on the square of the local WIMP abundance. In $[35,100]$ it was shown that the FermiLAT limits derived for WIMP annihilations into $W W$ (of which a large component of the total annihilation cross section should be comprised) are not yet sensitive to higgsino LSPs in the $100-350 \mathrm{GeV}$ range mainly due to the predicted under-abundance of neutralinos in these scenarios. Note that WIMPS having masses in the range $200 \mathrm{GeV}$ to a few $\mathrm{TeV}$ will be probed by the future CTA array [101, 102], i.e. any higgsino or wino-like LSP for which the relic abundance is within an order of magnitude of Planck would be seen. In [103] a comprehensive scan of the pMSSM was carried out, and DM limits (both present and projected) coming from the LHC, DD and ID experiments were studied (note that the collider study was not dedicated to the region of interest of the present paper; our optimisation of the kinematical cuts goes beyond previous analyses). Ref. [103] found an impressive degree of complementarity: in the range a few hundred $\mathrm{GeV}$ to $1 \mathrm{TeV}$ DD poses stronger bounds, and for higher masses ID is more sensitive. Therefore we conclude that for the mass range studied in this paper, the LHC and DD limits are the most relevant.

\section{LHC potential to probe NSUSY}

In the previous section we have discussed the current and future sensitivity of underground experiments to NSUSY scenario. In this section we explore the LHC potential to probe 

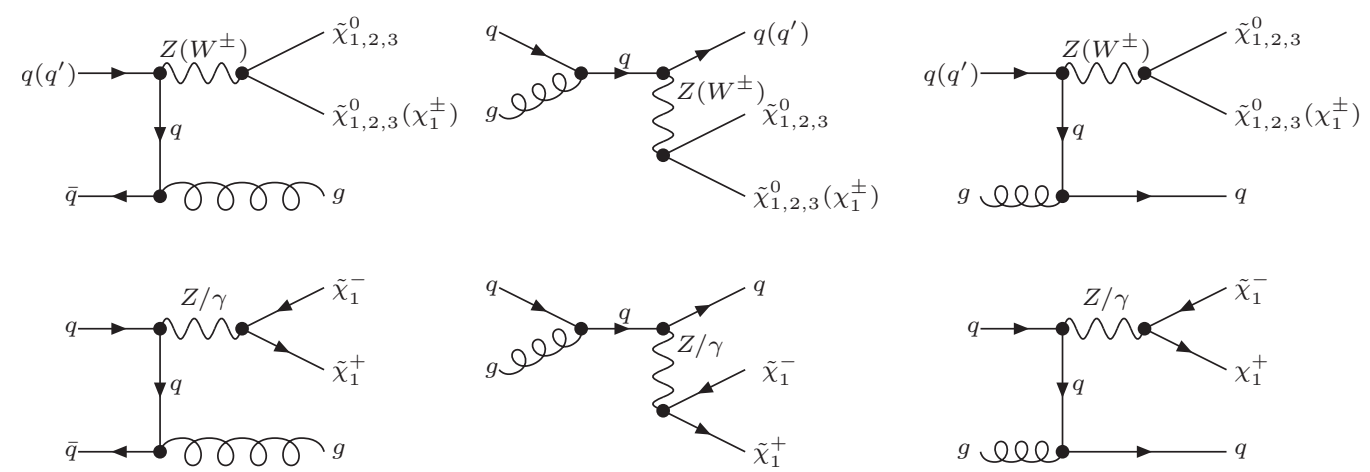

Figure 6. Representative diagrams for pair neutralino-chargino production in association with quark/gluon leading to monojet signature.

NSUSY and demonstrate that it plays a crucial complementary role.

The LHC's most sensitive searches for quasi-degenerate $\tilde{\chi}_{i}^{ \pm}$and $\tilde{\chi}_{i}^{0}$ scenario are monojet signatures, i.e. the production of a pair of electroweakinos through the s-channel exchange of a SM EW gauge boson, $Z, \gamma$ or $W^{ \pm}$, accompanied by hard QCD initial state radiation (ISR) via the process

$$
p p \rightarrow \chi_{a} \chi_{b} j \quad \chi_{a, b}=\tilde{\chi}_{1,2,3}^{0}, \tilde{\chi}_{1}^{ \pm} .
$$

The corresponding Feynman diagrams can be found in figure 6 .

As we discuss below, the main problem for the signal search in this channel is the large background, the dominant contribution coming from the EW production processes $Z+$ jets and $W+$ jets. We begin our analysis of the monojet signal from the recasting of the LHC at $\sqrt{s}=8 \mathrm{TeV}$ data and the respective experimental results, and then analyse the prospects of the $13 \mathrm{TeV}$ LHC run with both standard and high luminosity (HL) options. Both ATLAS [78] and CMS [104] have performed studies of monojet signatures at the LHC Run1, which have been interpreted in the context of an EFT approach. However this approach cannot be used for the NSUSY scenario because of the $Z, W, \gamma$ mediating interactions as indicated in figure 6. Hence, in the case of NSUSY,

$$
\text { DM with EW mediators } \not \mathcal{L}_{\text {eff }}=\frac{1}{\Lambda^{2}}(q \Gamma \bar{q})(\chi \bar{\chi})
$$

where $\Gamma$ is some Lorentz structure and $\chi$ is the DM particle. Therefore it is necessary to recast the searches in terms of this NSUSY scenario and not to use limits from $\Lambda$ $\mathrm{DM}_{\text {mass }}$ plane.

\subsection{Analysis setup}

In this section we describe the different aspects of our simulation of both the signal and the most important backgrounds, implementing the important steps of hadronization and fast detector simulation.

We performed a parton-level simulation using MadGraph v1.5.11 [105] with the MSSM model available on the FeynRules web page [106] implemented in UFO format [107], ${ }^{1}$

\footnotetext{
${ }^{1}$ http://feynrules.irmp.ucl.ac.be/wiki/MSSM
} 


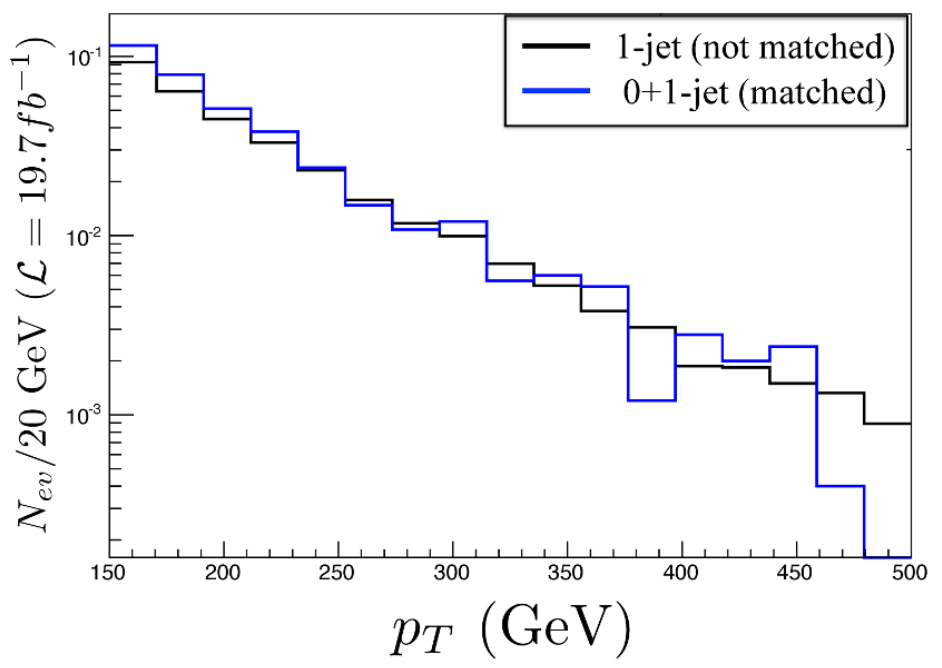

Figure 7. Leading jet $p_{T}$ cross section distributions for the case of the $0+1$ jet matched sample (blue) and 1 jet sample (black) for the $\tilde{\chi}_{1}^{0} \tilde{\chi}_{2}^{0}$ production at the $8 \mathrm{TeV}$ LHC.

and cross-checked results against CalcHEP [108] with the MSSM model from the HEPMDB website. ${ }^{2}$

At the level of matrix-element we have generated the production of a pair of electroweakinos via the s-channel exchange of a SM EW gauge bosons, accompanied by hard QCD initial state radiation. Parton level SM background simulations have been also cross checked between two packages. Our choice of PDF sets is CTEQ6L1 [109] and we used the MadGraph dynamical choice of renormalization scale which is equal to the geometric mean of $\mathrm{Mass}^{2}+\mathrm{P}_{\mathrm{T}}^{2}$ for the final state particles. Parton showering, hadronisation and decay of the unstable particles were simulated using PYTHIA v6.4 [110] while detector effects have been simulated with Delphes3 [111] employing a suitable CMS card. Finally, the background processes yields, which include $Z+j, W+j, t \bar{t}$, QCD and single top processes, have been taken from the experimental results for $8 \mathrm{TeV}$ analysis and has been simulated using MadGraph+PTYHIA+DELPHES chain for the $13 \mathrm{TeV}$ analysis.

For the signal and background events at the parton level we have applied a cut on the jet transverse momentum of $p_{T}^{j}>90 \mathrm{GeV}$ at the generation level. This is a subtle point. One might argue that, since dealing with processes involving QCD radiation, it is necessary to apply a merging procedure between the hard jet generated via the parton-level matrix element and the soft jets generated by the showering algorithm, merging therefore the 0-jet and 1-jet samples. However, since a hard jet is selected at the analysis level (e.g. $p_{T}^{j}>110 \mathrm{GeV}$ for the $8 \mathrm{TeV}$ LHC analysis and higher for the $13 \mathrm{TeV}$ case, with a final selection requirement of a high $E_{T}^{\text {miss }}$, somewhat correlated with the jet $p_{T}$ [112]) we found that this matching was unnecessary, and generated just the one jet sample. Moreover, avoiding matching in this case allowed us to make our analysis much more effective and obtain enough statistics in the high $p_{T}^{j}$ region. To illustrate the validity of this procedure,

\footnotetext{
${ }^{2}$ http://hepmdb.soton.ac.uk/hepmdb:0611.0028
} 
in figure 7 we present the $p_{T}$ distribution of the leading jet for the case of $\tilde{\chi}_{1}^{0} \tilde{\chi}_{2}^{0}$ production. Shown in blue is the leading jet $p_{T}$ for the case of a $0+1$ matched jet sample, while the case of the 1-jet events being unmatched is shown in black. One can indeed see that in case of a high $p_{T}$ cut on the leading jet at the analysis level, the matched and unmatched distributions of the leading jet are very similar, indicating that contribution from the 0 -jet matched sample is negligible, and that the $p_{T}$ of the leading jet is dominated by the one-jet sample in the high $p_{T}$ region.

\subsection{LHC Run1: the reach of monojet searches}

We start our analysis with the exploration of the $8 \mathrm{TeV}$ LHC potential to probe NSUSY and the recasting of the respective experimental results. For this purpose we have chosen CMS monojet analysis [104] which has been done for the data recorded at $\sqrt{s}=8 \mathrm{TeV}$ with $19.5 \mathrm{fb}^{-1}$ integrated luminosity. ${ }^{3}$ We have applied the following trigger selection followed by the cut-flow according to the CMS analysis:

- Two triggers which require $E_{T}^{\text {miss }}>120 \mathrm{GeV}$ or $E_{T}^{\text {miss }}>105 \mathrm{GeV}$ and a jet with $p_{T}>80 \mathrm{GeV}$ and within $|\eta|<2.6$.

- The analysis then requires that the jet with the highest transverse momenta has $p_{T}>110 \mathrm{GeV}$ and $|\eta|<2.4$.

- Events with more than two jet with $p_{T}>30 \mathrm{GeV}$ and $|\eta|<4.5$ are discarded together with events where $\Delta \phi\left(j_{1}, j_{2}\right)<2.5$, where $j_{1}$ and $j_{2}$ are the leading and sub-leading jets, to reduce QCD background.

- The $W$ production background was suppressed by applying a veto on events with one electron or muon satisfying $p_{T}>10 \mathrm{GeV}$ cut and events with one tau jet with $p_{T}>20 \mathrm{GeV}$ and $|\eta|<2.3$.

- Finally the analysis was performed in 7 regions with an increasing requirement of $E_{T}^{\text {miss }}: E_{T}^{\text {miss }}>250,300,350,400,450,500$ and $550 \mathrm{GeV}$.

We have then derived the signal significance for each signal region from the number of signal events (S), after having imposed the cuts above, and the number of background events (B), using the following expression as in the CMS analysis:

$$
\alpha=2(\sqrt{S+B}-\sqrt{B}),
$$

which is similar to the more common $S / \sqrt{S+B}$ for $S \ll B$, but is more robust with respect to downward fluctuations [114].

In figure 8 we show contours of iso-significance $\alpha$ (solid gray) in the $\mu-M_{1}$ plane for the lowest (left frame) and highest (right frame) requirements on $E_{T}^{\text {miss }}$. The LUX exclusion is further shown by the green shaded area as well as the LEP2 limit on charginos by the red shaded area. In addition, the $\mathrm{S} / \mathrm{B}$ ratio is shown by a blue-dashed line. By only inspecting

\footnotetext{
${ }^{3}$ The respective ATLAS analysis, which leads to similar results, can be found in [113].
} 

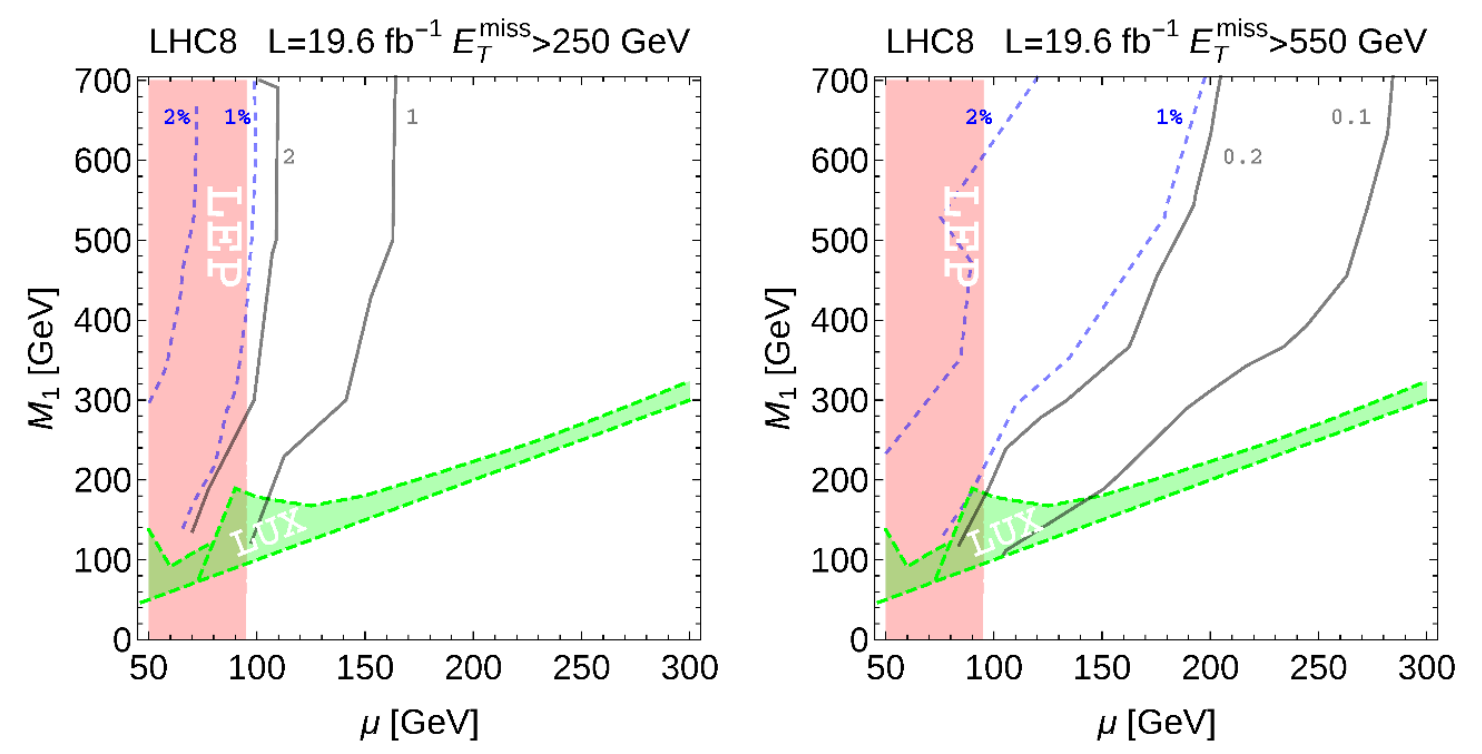

Figure 8. Contours for the $2(\sqrt{S+B}-\sqrt{B})$ (gray) and S/B (blue-dashed)in the plane $\left(\mu, M_{1}\right)$ for the signal regions 1 and 7 as defined in [104]. LUX and LEP exclusions are shown in green and red.

significance contours, one could think that the Run1 LHC data could slightly extend the LEP2 limit using the low $E_{T}^{\text {miss }}$ signal region: the area on the left of the lines of $\alpha=2$ would be ruled out at $95 \%$ confidence level (CL). However, one then observes that the $S / B$ never goes above $2 \%$ in the region allowed by LEP 2 , while the actual systematic uncertainties of this analysis are of the order of $5-10 \%[78,104]$. This means that the low $S / B$ ratio for the $8 \mathrm{TeV}$ LHC is the main obstacle to going beyond the LEP2 limits. We can also see from figure 8 (right) that the higher $E_{T}^{\text {miss }}$ cut increases the $\mathrm{S} / \mathrm{B}$ ratio, eventually at the expense of the signal, meaning that the significance drops below $\alpha=2$ level in the parameter space allowed by LEP2. Therefore we conclude that Run1 LHC does not have the potential to test the NSUSY scenario beyond the LEP2 limit due to systematic uncertainties.

\section{3 $13 \mathrm{TeV}$ LHC potential and complementarity to underground experiments}

As we have seen in the previous section, the LHC Run1 is not sensitive to the NSUSY parameter space we consider due to the low statistical significance and the fact that the low $\mathrm{S} / \mathrm{B}$ ratio remains below the systematic errors.

In this section we study the LHC Run2 case and show that the higher collider luminosity and energy allow us to choose kinematical cuts, bringing the S/B ratio to a desirable level while keeping the statistical significance at a high enough level in order to establish sensitivity to the NSUSY parameter space. Being a very challenging scenario, we make projections up to HL configuration of the LHC machine for different assumptions regarding the $E_{T}^{\text {miss }}$ cuts and the control over systematic uncertainties.

In order to ensure that the S/B ratio is under control, we compare the relative size and shape difference of the signal versus the dominant irreducible background $Z+j e t \rightarrow \nu \bar{\nu}+j e t$ $(Z j)$. The relevant parton level distribution are shown as a function of the jet $p_{T}$ in figure 9 . 

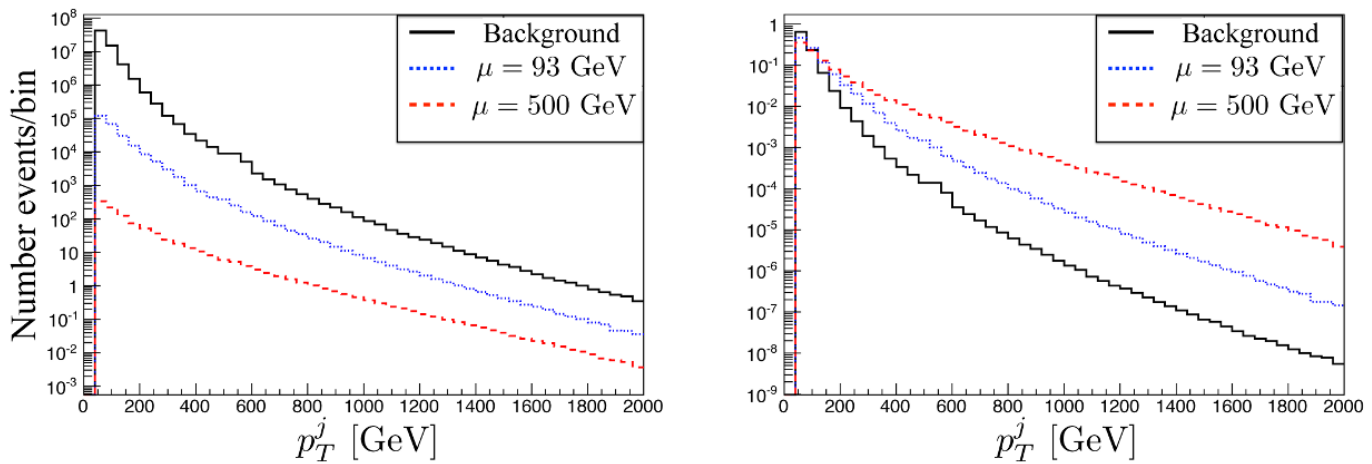

Figure 9. Signal (dotted blue and dashed red) and $Z j$ background (solid black) parton-level $p_{T}^{j}$ distributions for the $13 \mathrm{TeV}$ LHC for the NSUSY scenario. Left: $p_{T}^{j}$ distributions for $100 \mathrm{fb}^{-1}$ integrated luminosity. Right: normalised signal and $Z j$ background distributions.

One can see that even for $\mu=93 \mathrm{GeV}$ corresponding to $m_{\chi_{1}^{0}} \simeq 100 \mathrm{GeV}$, the background is about 3 orders of magnitude higher than the signal for a low $p_{T}^{j}>$ cut. An important feature of the signal versus background is that the shape of the background distribution is quite different from the signal: the background falls more rapidly with $p_{T}^{j}$, and the difference in the slope with respect to the signal is bigger for higher neutralino masses. The different in slope is mainly due to the mass difference between the neutralino, from the signal, and the neutrino, from the background. One should also notice that the difference between the shapes of the signal and background $p_{T}^{j}$ distributions vanishes for very large values of $p_{T}^{j} \gg m_{\chi_{1}^{0}}$, as one would expect. This provides us with our main strategy to optimise the LHC sensitivity to the NSUSY parameter space, that is to find the maximal value for $E_{T}^{\text {miss }}$ cut while maintaining the statistical significance at high enough level. On inspecting this figure one can already see that in order to achieve S/B ratio at about $5 \%$ level the value $E_{T}^{\text {miss }}$ cut should be around of $1 \mathrm{TeV}$.

The signal process analysed is the same as that in eq. (4.1), with the only difference being that the initial requirement on the QCD ISR has been increased to $150 \mathrm{GeV}$, motivated by our preliminary study in ref. [112].

Along with the signal processes, we have simulated the two main backgrounds

$$
\begin{aligned}
& p p \rightarrow Z j \rightarrow \nu_{l} \bar{\nu}_{l} j \\
& p p \rightarrow W j \rightarrow l^{+} \nu_{l}+\text { c.c. }
\end{aligned}
$$

with $l=e, \mu, \tau$.

Inspired by the $8 \mathrm{TeV}$ CMS monojet search we have then applied the following cut-flow

- We require a leading jet with $p_{T}>200 \mathrm{GeV}$ and $|\eta|<2.4$

- We apply a veto on events with more than two jets with $p_{T}>30 \mathrm{GeV}$ and $|\eta|<4.5$

- We require $\Delta \phi\left(j_{1}, j_{2}\right)<2.5$

- We apply a veto on electrons and muons with $p_{T}>10 \mathrm{GeV}$.

- We apply a veto on taus with $p_{T}>20 \mathrm{GeV}$ and $|\eta|<2.3$. 


\begin{tabular}{|c|c|c|c|c|}
\hline & $Z j, Z \rightarrow \nu \nu$ & $W j, W \rightarrow l \nu$ & $\begin{array}{c}\mu=100 \mathrm{GeV} \\
M_{1}=700 \mathrm{GeV}\end{array}$ & $\begin{array}{c}\mu=200 \mathrm{GeV} \\
M_{1}=800 \mathrm{GeV}\end{array}$ \\
\hline Initial \# of events & $3.15 \cdot 10^{6}$ & $1.25 \cdot 10^{7}$ & $3.63 \cdot 10^{5}$ & $6.45 \cdot 10^{3}$ \\
\hline$p_{T}^{j}>200 \mathrm{GeV}\left|\eta^{j}\right|<2.4$ & $1.05 \cdot 10^{6}$ & $4.11 \cdot 10^{6}$ & $1.73 \cdot 10^{5}$ & 3528 \\
Jet veto & $8.7 \cdot 10^{5}$ & $3.13 \cdot 10^{6}$ & $1.33 \cdot 10^{5}$ & 2691 \\
$\Delta \phi\left(j_{1}, j_{2}\right)<2.5$ & $7.2 \cdot 10^{5}$ & $2.3 \cdot 10^{6}$ & $1.10 \cdot 10^{5}$ & 2320 \\
Veto $e^{ \pm}, \mu^{ \pm}, \tau^{ \pm}$ & $7.2 \cdot 10^{5}$ & $6.8 \cdot 10^{5}$ & $1.08 \cdot 10^{5}$ & 2301 \\
$E_{T}^{\text {miss }}>200 \mathrm{GeV}$ & $6.4 \cdot 10^{5}$ & $4.3 \cdot 10^{5}$ & 9846 & 2188 \\
$E_{T}^{\text {miss }}>600 \mathrm{GeV}$ & 4353 & 1002 & 171 & 93 \\
$E_{T}^{\text {miss }}>700 \mathrm{GeV}$ & 1703 & 250 & 80 & 47 \\
$E_{T}^{\text {miss }}>800 \mathrm{GeV}$ & 694 & 0 & 37 & 22 \\
\hline
\end{tabular}

Table 1. Cutflow for the two main SM background and two choices of signal for the $13 \mathrm{TeV}$ LHC with $100 \mathrm{fb}^{-1}$ of integrated luminosity. The initial number of events corresponds to $p_{T}^{j}>$ $150 \mathrm{GeV}$ cut.
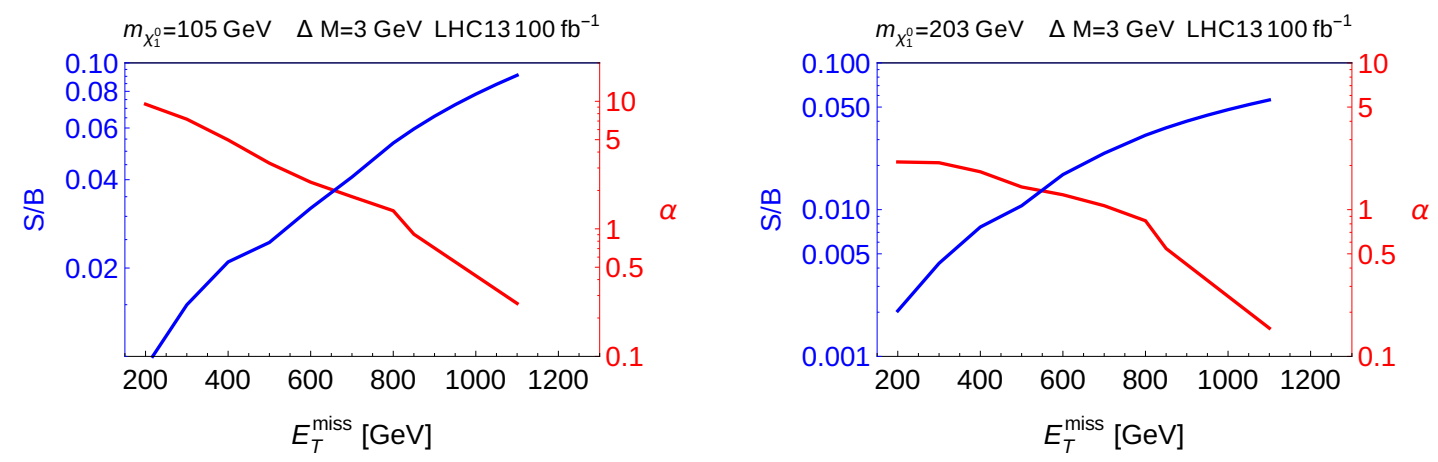

Figure 10. S/B and $\alpha$ as a function on the final selection cut on the $E_{T}^{\text {miss }}$ for $\tilde{\chi}_{1}^{0} \sim 100 \mathrm{GeV}$ (left panel) and $\sim 200 \mathrm{GeV}$ (right panel).

We have then defined signal regions with increasing cuts on $E_{T}^{\text {miss }}$; an example cut flow is provided in table 1 . We have not generated $t \bar{t}$, QCD and single top background, though we have applied to our simulated samples cuts that reduce these background to a negligible level with respect to $W j$ and $Z j$, see details in refs. [78, 104].

As observed for the case of $8 \mathrm{TeV}$ LHC, a strong tension arises in attempting to simultaneous maximise the $\mathrm{S} / \mathrm{B}$ ratio and the statistical significance. We demonstrate this in figure 10, where we plot $\mathrm{S} / \mathrm{B}$ and $\alpha$ as a function on the final selection cut on the $E_{T}^{\text {miss }}$ for both the case of $\tilde{\chi}_{1}^{0} \sim 100 \mathrm{GeV}$ (left panel) and $\sim 200 \mathrm{GeV}$ (right panel). This figure clearly indicates that in order to achieve a high enough S/B ratio in keeping with the expected level of systematic uncertainties, a hard cut on $E_{T}^{\text {miss }}$ ought to be applied, which at the same time pulls the significance down, due to the reduction in the number of signal events.

Given this tension it is therefore important to optimize the $E_{T}^{\text {miss }}$ cut to provide a high enough $\mathrm{S} / \mathrm{B}$ ratio and to keep $\alpha>2$ (5) in order to obtain an exclusion (discovery). The 


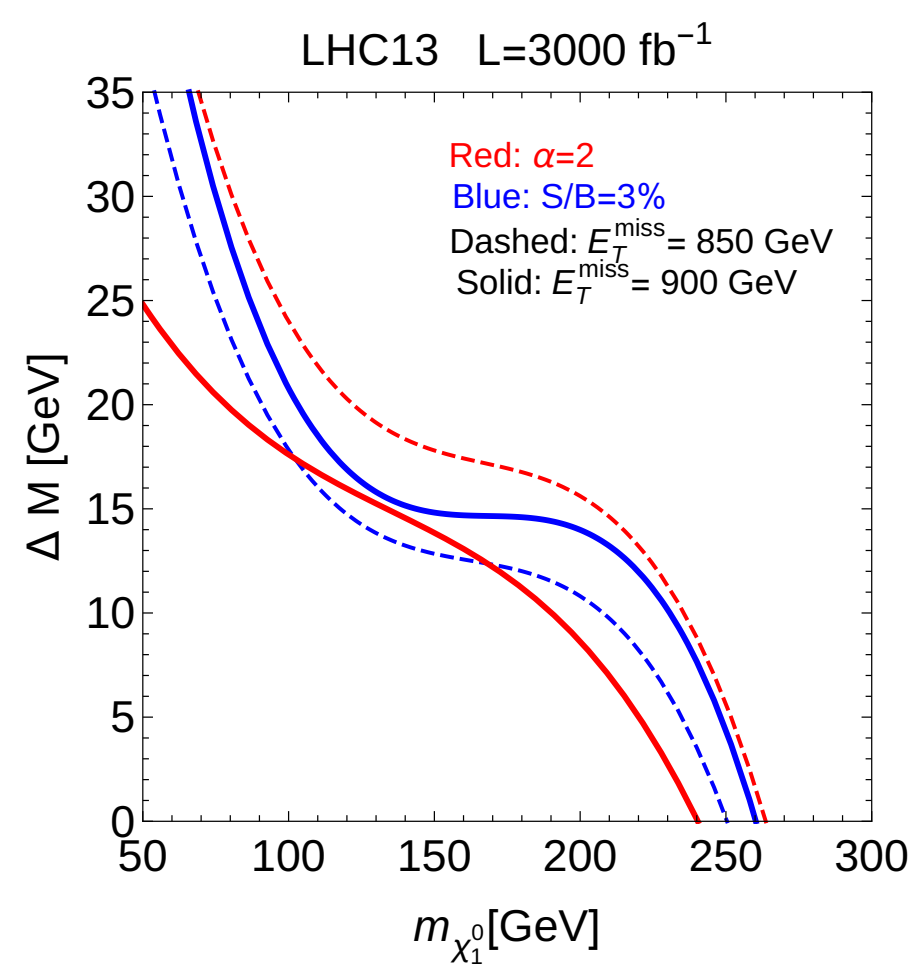

Figure 11. S/B (blue) and $\alpha$ (red) isocontours for two choices of $E_{T}^{\text {miss }}$ cut: $850 \mathrm{GeV}$ (dashed) and $900 \mathrm{GeV}$ (solid) in the $m_{\chi_{1}^{0}}-\Delta \mathrm{M}$ plane.

NSUSY parameter space can be conveniently described and presented in $m_{\tilde{\chi}_{1}^{0}}-\Delta \mathrm{M}$ plane, where for $\Delta \mathrm{M}$ we have chosen the mass difference between the lightest chargino and the DM particle. In this plane, for a given value of integrated luminosity, the optimal $E_{T}^{\text {miss }}$ cut can be chosen by the point where S/B and $\alpha$ cross or are as close to each other as possible. This is related to the fact that the iso-significance contours are shifted to the left in the $m_{\tilde{\chi}_{1}^{0}}-\Delta \mathrm{M}$ plane with the increase in the $E_{T}^{\text {miss }}$ cut due to the decrease of signal statistics, while iso-S/B contours are shifted to the right at the same time due to the increase of S/B ratio. Therefore the case when the respective iso-contours cross/are close to each other, would provide the maximal exclusion or discovery area in the $m_{\tilde{\chi}_{1}^{0}}-\Delta M$ plane.

We illustrate this in figure 11 which presents $\mathrm{S} / \mathrm{B}$ and significance isocontours in the $m_{\tilde{\chi}_{1}^{0}} \Delta \mathrm{M}$ plane for two different cuts on $E_{T}^{\mathrm{miss}}, 850$ and $900 \mathrm{GeV}$. One can see that indeed for $E_{T}^{\text {miss }}>850 \mathrm{GeV}$, the exclusion area is below $S / B=3 \%$ (blue dashed) contour, while for $E_{T}^{\text {miss }}>900 \mathrm{GeV}$, the area below $\alpha=2$ (red solid) contour is excluded. Since for the first case the exclusion area is bigger, the $E_{T}^{\text {miss }}>850 \mathrm{GeV}$ is better choice for the optimal cut. We have found that a cut around 600 (850) GeV for $100 \mathrm{fb}^{-1}\left(3000 \mathrm{fb}^{-1}\right)$ provides $\alpha \simeq 2$ and $S / B \simeq 0.03$ iso-contours optimally close to each other, which maximises the reach of the $13 \mathrm{TeV}$ LHC for the NSUSY parameter space. The proximity of $\alpha \simeq 2$ and $S / B \simeq 0.05$ iso-contours eventually requires higher $E_{T}^{\text {miss }}$ cut which is found to be around $950 \mathrm{GeV}$, and as a result leads to a poorer $13 \mathrm{TeV}$ LHC reach as we discuss below. While presenting results in the $m_{\tilde{\chi}_{1}^{0}}-\Delta M$ plane, we separate the cases $M_{1}>0$ and $M_{1}<0$, which differ due to different bino-higgsino components and mass splittings as discussed earlier, which eventually becomes less and less relevant as we approach the low $\Delta \mathrm{M}$ region. 

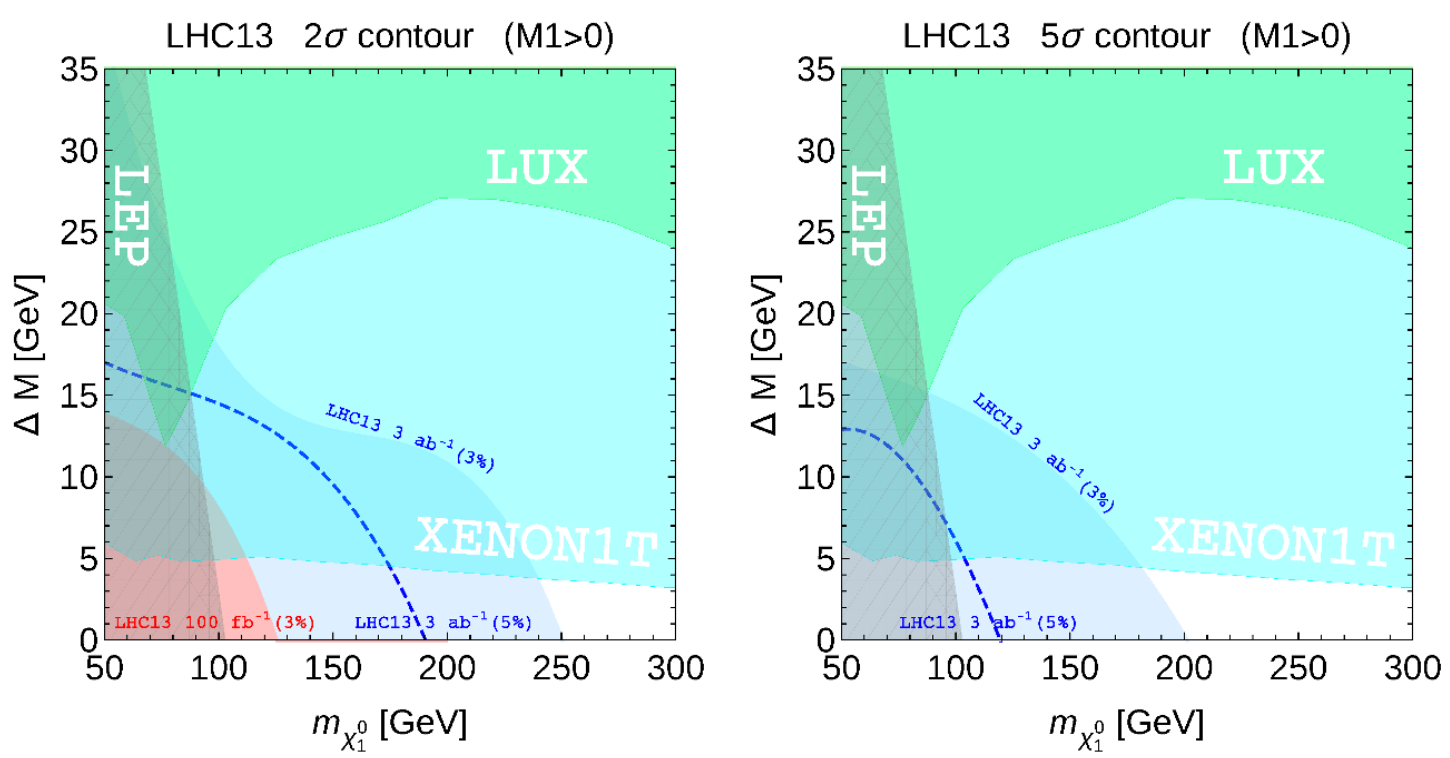

Figure 12. Exclusion (left) and discovery (right) contour lines for the $13 \mathrm{TeV}$ LHC at the end of the LHC Run2 (light red region) and of the HL-LHC (light blue region) assuming S/B $>3 \%$. For the latter case also the case $\mathrm{S} / \mathrm{B}>5 \%$ is shown. The region excluded by LUX and the projected exclusion by XENON1T are also shown, together with the LEP limit on the $\tilde{\chi}_{1}^{ \pm}$mass. $M_{1}>\mu$ is considered here.

In figure 12 and figure 13 we show results for $M_{1}>\mu$ and $M_{1}<-\mu$ (note that we have chosen $\mu>0$ ). The left panels contain the $2 \sigma$ exclusion LHC reach while the right panels contain the $5 \sigma$ discovery LHC potential. Both the cases of requiring $3 \%$ and $5 \%$ for the S/B ratio are shown, the latter just for the High Luminosity (HL)-LHC scenario. In the same plot we present also LUX sensitivity and the projected sensitivity of XENON1T to the in the $m_{\tilde{\chi}_{1}^{0}} \Delta M$ plane. One can see that at Run2, with $\sim 100 \mathrm{fb}^{-1}$ of integrated luminosity collected, the LHC will be able to exclude up to $\sim 150 \mathrm{GeV} \tilde{\chi}_{1}^{0}$ with $\Delta M$ below $5 \mathrm{GeV}$, if the systematic error can be kept at the $3 \%$ level. The LHC will therefore surpass the LEP sensitivity for this scenario which reached the limit $\tilde{\chi}_{1}^{ \pm}>103 \mathrm{GeV}$ for low $\Delta \mathrm{M}$ values as shown in figure 12 and figure 13 . By the end of the HL-LHC run, up to $\sim 250$ (200) GeV LSP could be excluded for low mass splitting with a $\mathrm{S} / \mathrm{B}>3 \%$ (5\%). It is quite remarkable that the LHC has the maximal sensitivity in the low $\Delta \mathrm{M}$ region. This reach is nicely complemented by the LUX results and the projected exclusions for XENON1T, which cover the region with higher mass splitting. Such complementarity would allow LHC and DD experiments to completely exclude NSUSY scenario with DM mass up to $\sim 250 \mathrm{GeV}$. Discovery prospects for this scenario are shown in the right panel of figure 12 which demonstrates that $\sim 180(110) \mathrm{GeV} \chi_{1}^{0}$ can be discovered for $\mathrm{S} / \mathrm{B}>5 \%(3 \%)$ at the end of the HL-LHC run, while with $100 \mathrm{fb}^{-1}$ the LHC will not have discovery sensitivity.

At the end of this section we would like to discuss a potentially promising new signature involving a monojet plus soft di-leptons which was studied in terms of the NSUSY parameter space in ref. [76]. In short, in this paper the signal from the second neutralino decaying leptonically into the lightest neutralino has been studied. It was suggested that 

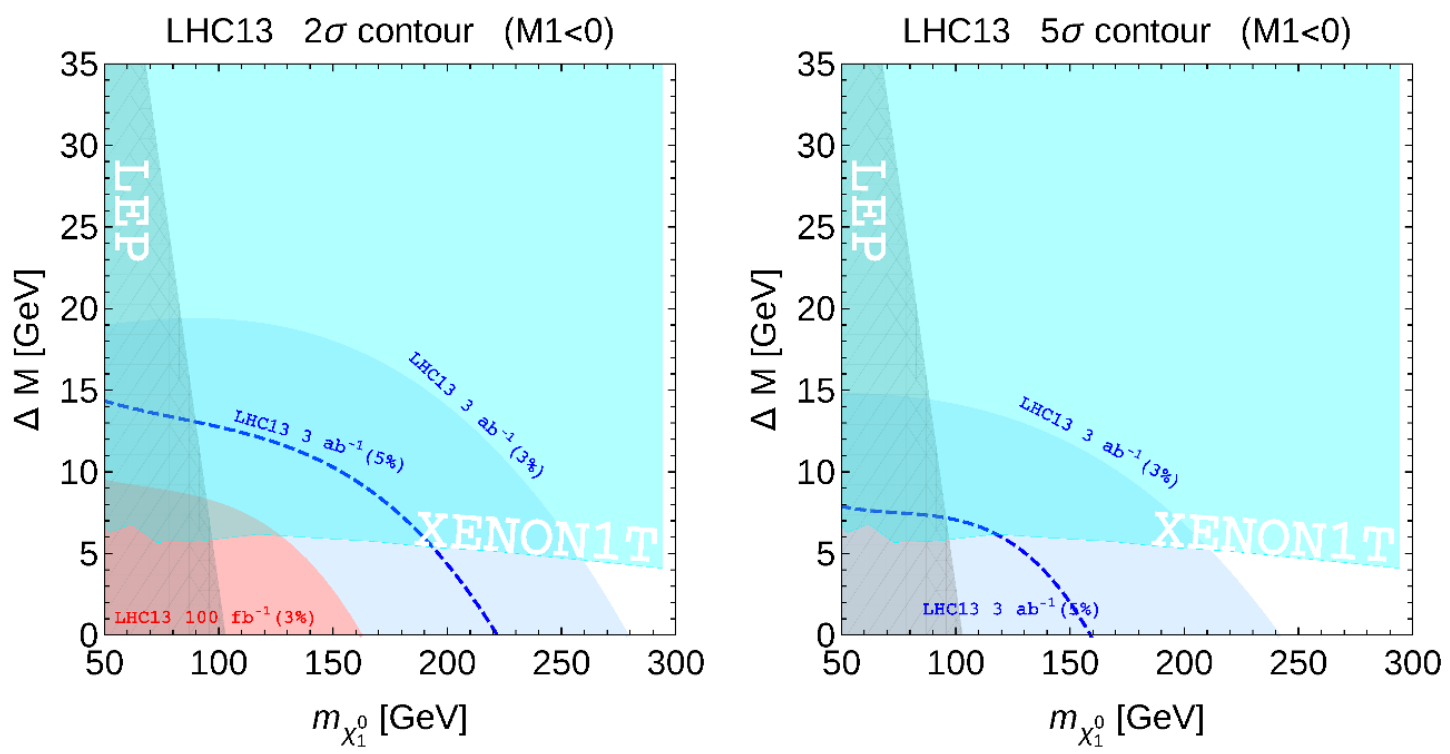

Figure 13. Exclusion (left) and discovery (right) contour lines for the $13 \mathrm{TeV}$ LHC at the end of the LHC Run2 (light red region) and of the HL-LHC (light blue region) assuming S/B $>3 \%$. For the latter case also the case $\mathrm{S} / \mathrm{B}>5 \%$ is shown. The region excluded by LUX and the projected exclusion by XENON1T are also shown, together with the LEP limit on the $\tilde{\chi}_{1}^{ \pm}$mass. $M_{1}<-\mu$ is considered here.

one could trigger on soft leptons and use the upper cut on di-lepton invariant mass below $10 \mathrm{GeV}$ to suppress background and extract the signal from compressed $\tilde{\chi}_{1}^{0}-\tilde{\chi}_{2}^{0}$ production. After the suggested cuts, the background can be reduced down to about the $6 \mathrm{fb}$ level bringing it below the signal, which would allow one to claim a discovery for chosen benchmarks with only $100 \mathrm{fb}^{-1}$ of integrated luminosity. One should note however, that in spite of the comprehensive set of backgrounds, $j b \bar{b}$ was not considered in this paper. After application of the cuts from [76] we have found that $j b \bar{b}$ background is in fact dominant for the monojet plus soft di-lepton signal and is about two orders of magnitude above the backgrounds taken into account in the aforementioned study. An estimation of $j b \bar{b}$ background is not trivial, since the cross section of the $j b \bar{b}$ process is very high, about $100 \mathrm{nb}$ for soft initial cuts on jet $p_{T}$, while the efficiency of the selection cuts is very low, about $10^{-5}$. Therefore, a reasonable estimate of this background requires either the simulation of at least $10^{6}$ events or the extraction of this background from future experimental data. Our preliminary results indicate that one should develop a dedicated strategy to suppress this $j b \bar{b}$ background in order to make the monojet plus soft di-lepton signal a viable tool for the exploration of the NSUSY parameter space.

\section{Conclusions}

In this paper we have explored the complementary potential of the Large Hadron Collider and underground experiments to probe Dark Matter (DM) in the Natural Supersymmetry (NSUSY) scenario. This study, which combines searches from different kinds of experiments, has to be done in the context of a specific model, as (model-independent) Effective 
Theory (EFT) approaches are very limited in scope, see e.g. the discussion in refs. $[115,116]$. In particular the EFT approach is not applicable for well motivated NSUSY scenario, which we study here, where DM has direct couplings to Standard Model electroweak (EW) gauge bosons and the Higgs.

Current limits on simple SUSY scenarios are at the TeV range, in clear tension with naturalness arguments and hence with the motivation for introducing SUSY in the first place. A possible explanation for this situation is that the manifestation of SUSY is not as simple as one expects, but there is more complexity in the structure of SUSY at highenergies. Notwithstanding, one would still expect that the particles more directly related to the tuning of the EW scale remain light in the spectrum. This leads to a generic expectation that DM in NSUSY should have a sizeable Higgsino component.

While being theoretically attractive this scenario also represents a clear example of how colliders and underground experiments can complement each other. Indeed, while underground experiments have a larger mass sensitivity than colliders, being able to probe the multi- $\mathrm{TeV}$ region, colliders can cover parameter space hidden from DM direct detection (DD) experiments. Specifically, the increase of the DM higgsino component makes NSUSY parameter space increasingly difficult to probe in DD experiments. In this region higgsinolike DM is quasi-degenerate with two other particles, the second neutralino and the lightest chargino, and the increase of higgsino component is correlated with the decrease of this mass splitting, $\Delta \mathrm{M}$. At the same time, the LHC sensitivity increases with the increase of the higgsino component of DM and reaches its maximum for very low values of $\Delta \mathrm{M}$. We have conveniently described the NSUSY parameter space in the $\mu-M_{1}$ region and have translated it into sensitivity of the LHC and DM DD experiments in the $m_{\tilde{\chi}_{1}^{0}}-\Delta \mathrm{M}$ plane, where for $\Delta \mathrm{M}$ we have chosen the mass difference between the lightest chargino and DM. We have studied the current and the future reach of underground experiments in this region, as well as given predictions for the relic abundance in NSUSY.

We would like to point out that we have presented combined LHC and DM DD experiments results for the whole $m_{\tilde{\chi}_{1}^{0}} \Delta \mathrm{M}$ NSUSY space, rather than for chosen benchmarks. Moreover, we have optimised the final $E_{T}^{\text {miss }}$ selection cut to keep the S/B ratio at a high enough level, to deal with the systematic errors on the background, which aer one of the main problem for the exploration of the NSUSY parameter space. As a result, we have found that the $8 \mathrm{TeV}$ LHC unfortunately does not allow to test the NSUSY parameter space beyond the LEP2 limits, while the $13 \mathrm{TeV}$ LHC at $3 \mathrm{ab}^{-1}$ has the potential to significantly surpass the LEP2 reach and to cover DM masses up to about $250 \mathrm{GeV}$ for $\Delta \mathrm{M}<5 \mathrm{GeV}$, which can neither be covered by LUX nor by XENON1T DM DD experiments. At the same time the XENON1T experiment will be able to complementarily cover the $\Delta \mathrm{M}>5 \mathrm{GeV}$ parameter space up to large values of the DM mass, well beyond the LHC reach via monojet analyses.

\section{Acknowledgments}

The work of VS and AB is supported by the Science Technology and Facilities Council (STFC) under grant number ST/L000504/1 and ST/L000296/1 respectively. WP is sup- 
ported by the Bundesministerium für Bildung und Forschung (BMBF) under contract no. 05H12WWE. AKMB thanks Andreas Goudelis for helpful discussions.

Open Access. This article is distributed under the terms of the Creative Commons Attribution License (CC-BY 4.0), which permits any use, distribution and reproduction in any medium, provided the original author(s) and source are credited.

\section{References}

[1] J.R. Ellis, K. Enqvist, D.V. Nanopoulos and F. Zwirner, Observables in low-energy superstring models, Mod. Phys. Lett. A 1 (1986) 57 [INSPIRE].

[2] R. Barbieri and G.F. Giudice, Upper bounds on supersymmetric particle masses, Nucl. Phys. B 306 (1988) 63 [INSPIRE].

[3] G.L. Kane, C.F. Kolda, L. Roszkowski and J.D. Wells, Study of constrained minimal supersymmetry, Phys. Rev. D 49 (1994) 6173 [hep-ph/9312272] [INSPIRE].

[4] G.W. Anderson and D.J. Castano, Naturalness and superpartner masses or when to give up on weak scale supersymmetry, Phys. Rev. D 52 (1995) 1693 [hep-ph/9412322] [INSPIRE].

[5] S. Dimopoulos and G.F. Giudice, Naturalness constraints in supersymmetric theories with nonuniversal soft terms, Phys. Lett. B 357 (1995) 573 [hep-ph/9507282] [INSPIRE].

[6] K.L. Chan, U. Chattopadhyay and P. Nath, Naturalness, weak scale supersymmetry and the prospect for the observation of supersymmetry at the Tevatron and at the CERN LHC, Phys. Rev. D 58 (1998) 096004 [hep-ph/9710473] [INSPIRE].

[7] S. Akula, M. Liu, P. Nath and G. Peim, Naturalness, supersymmetry and implications for LHC and dark matter, Phys. Lett. B 709 (2012) 192 [arXiv:1111.4589] [INSPIRE].

[8] M. Liu and P. Nath, Higgs boson mass, proton decay, naturalness and constraints of the LHC and Planck data, Phys. Rev. D 87 (2013) 095012 [arXiv: 1303.7472] [InSPIRE].

[9] P.H. Chankowski, J.R. Ellis and S. Pokorski, The fine tuning price of LEP, Phys. Lett. B 423 (1998) 327 [hep-ph/9712234] [INSPIRE].

[10] P.H. Chankowski, J.R. Ellis, M. Olechowski and S. Pokorski, Haggling over the fine tuning price of LEP, Nucl. Phys. B 544 (1999) 39 [hep-ph/9808275] [INSPIRE].

[11] R. Barbieri and A. Strumia, About the fine tuning price of LEP, Phys. Lett. B 433 (1998) 63 [hep-ph/9801353] [INSPIRE].

[12] G.L. Kane and S.F. King, Naturalness implications of LEP results, Phys. Lett. B 451 (1999) 113 [hep-ph/9810374] [INSPIRE].

[13] M. Bastero-Gil, G.L. Kane and S.F. King, Fine tuning constraints on supergravity models, Phys. Lett. B 474 (2000) 103 [hep-ph/9910506] [INSPIRE].

[14] J.L. Feng, K.T. Matchev and T. Moroi, Focus points and naturalness in supersymmetry, Phys. Rev. D 61 (2000) 075005 [hep-ph/9909334] [INSPIRE].

[15] J.L. Feng, K.T. Matchev and T. Moroi, Multi-TeV scalars are natural in minimal supergravity, Phys. Rev. Lett. 84 (2000) 2322 [hep-ph/9908309] [INSPIRE].

[16] J.L. Feng and D. Sanford, A natural 125 GeV Higgs Boson in the MSSM from focus point supersymmetry with A-terms, Phys. Rev. D 86 (2012) 055015 [arXiv:1205.2372] [INSPIRE]. 
[17] J.L. Feng, Naturalness and the status of supersymmetry, Ann. Rev. Nucl. Part. Sci. 63 (2013) 351 [arXiv: 1302.6587] [inSPIRE].

[18] J.A. Casas, J.R. Espinosa and I. Hidalgo, The MSSM fine tuning problem: a way out, JHEP 01 (2004) 008 [hep-ph/0310137] [INSPIRE].

[19] Y. Nomura and B. Tweedie, The supersymmetric fine-tuning problem and TeV-scale exotic scalars, Phys. Rev. D 72 (2005) 015006 [hep-ph/0504246] [INSPIRE].

[20] Y. Nomura, D. Poland and B. Tweedie, Minimally fine-tuned supersymmetric standard models with intermediate-scale supersymmetry breaking, Nucl. Phys. B $\mathbf{7 4 5}$ (2006) 29 [hep-ph/0509243] [INSPIRE].

[21] R. Kitano and Y. Nomura, Supersymmetry, naturalness and signatures at the LHC, Phys. Rev. D 73 (2006) 095004 [hep-ph/0602096] [INSPIRE].

[22] S. Cassel, D.M. Ghilencea and G.G. Ross, Fine tuning as an indication of physics beyond the MSSM, Nucl. Phys. B 825 (2010) 203 [arXiv:0903.1115] [InSPIRE].

[23] S. Cassel, D.M. Ghilencea and G.G. Ross, Testing SUSY at the LHC: electroweak and dark matter fine tuning at two-loop order, Nucl. Phys. B 835 (2010) 110 [arXiv:1001.3884] [INSPIRE].

[24] C. Brust, A. Katz, S. Lawrence and R. Sundrum, SUSY, the third generation and the LHC, JHEP 03 (2012) 103 [arXiv:1110.6670] [INSPIRE].

[25] M. Papucci, J.T. Ruderman and A. Weiler, Natural SUSY endures, JHEP 09 (2012) 035 [arXiv:1110.6926] [INSPIRE].

[26] L.J. Hall, D. Pinner and J.T. Ruderman, A natural SUSY Higgs near 126 GeV, JHEP 04 (2012) 131 [arXiv: 1112.2703] [inSPIRE].

[27] K. Blum, R.T. D'Agnolo and J. Fan, Natural SUSY predicts: Higgs couplings, JHEP 01 (2013) 057 [arXiv : 1206. 5303] [inSPIRE].

[28] J.R. Espinosa, C. Grojean, V. Sanz and M. Trott, NSUSY fits, JHEP 12 (2012) 077 [arXiv:1207.7355] [INSPIRE].

[29] R.T. D'Agnolo, E. Kuflik and M. Zanetti, Fitting the Higgs to natural SUSY, JHEP 03 (2013) 043 [arXiv: 1212.1165] [inSPIRE].

[30] H. Baer, V. Barger, P. Huang and X. Tata, Natural supersymmetry: LHC, dark matter and ILC searches, JHEP 05 (2012) 109 [arXiv:1203.5539] [INSPIRE].

[31] H. Baer, V. Barger, P. Huang, A. Mustafayev and X. Tata, Radiative natural SUSY with a 125 GeV Higgs boson, Phys. Rev. Lett. 109 (2012) 161802 [arXiv:1207.3343] [INSPIRE].

[32] H. Baer et al., Radiative natural supersymmetry: Reconciling electroweak fine-tuning and the Higgs boson mass, Phys. Rev. D 87 (2013) 115028 [arXiv: 1212.2655] [INSPIRE].

[33] H. Baer, V. Barger and M. Padeffke-Kirkland, Electroweak versus high scale finetuning in the 19-parameter SUGRA model, Phys. Rev. D 88 (2013) 055026 [arXiv:1304.6732] [INSPIRE].

[34] H. Baer, V. Barger, M. Padeffke-Kirkland and X. Tata, Naturalness implies intra-generational degeneracy for decoupled squarks and sleptons, Phys. Rev. D 89 (2014) 037701 [arXiv: 1311.4587] [INSPIRE]. 
[35] H. Baer, V. Barger and D. Mickelson, Direct and indirect detection of higgsino-like WIMPs: concluding the story of electroweak naturalness, Phys. Lett. B 726 (2013) 330 [arXiv:1303.3816] [INSPIRE].

[36] H. Baer, V. Barger, D. Mickelson and M. Padeffke-Kirkland, SUSY models under siege: LHC constraints and electroweak fine-tuning, Phys. Rev. D 89 (2014) 115019 [arXiv: 1404.2277] [INSPIRE].

[37] J.E. Younkin and S.P. Martin, Non-universal gaugino masses, the supersymmetric little hierarchy problem and dark matter, Phys. Rev. D 85 (2012) 055028 [arXiv:1201.2989] [INSPIRE].

[38] S. Fichet, Quantified naturalness from Bayesian statistics, Phys. Rev. D 86 (2012) 125029 [arXiv:1204.4940] [INSPIRE].

[39] G.D. Kribs, A. Martin and A. Menon, Natural supersymmetry and implications for Higgs physics, Phys. Rev. D 88 (2013) 035025 [arXiv:1305.1313] [INSPIRE].

[40] E. Hardy, Is natural SUSY natural?, JHEP 10 (2013) 133 [arXiv:1306.1534] [InSPIRE].

[41] K. Kowalska and E.M. Sessolo, Natural MSSM after the LHC 8 TeV run, Phys. Rev. D 88 (2013) 075001 [arXiv:1307.5790] [InSPIRE].

[42] K. Kowalska, L. Roszkowski, E.M. Sessolo and S. Trojanowski, Low fine tuning in the MSSM with higgsino dark matter and unification constraints, JHEP 04 (2014) 166 [arXiv: 1402.1328] [INSPIRE].

[43] C. Han, K.-i. Hikasa, L. Wu, J.M. Yang and Y. Zhang, Current experimental bounds on stop mass in natural SUSY, JHEP 10 (2013) 216 [arXiv:1308.5307] [INSPIRE].

[44] E. Dudas, G. von Gersdorff, S. Pokorski and R. Ziegler, Linking natural supersymmetry to flavour physics, JHEP 01 (2014) 117 [arXiv: 1308.1090] [INSPIRE].

[45] A. Arvanitaki, M. Baryakhtar, X. Huang, K. van Tilburg and G. Villadoro, The last vestiges of naturalness, JHEP 03 (2014) 022 [arXiv: 1309.3568] [INSPIRE].

[46] S.P. Martin, Nonuniversal gaugino masses and seminatural supersymmetry in view of the Higgs boson discovery, Phys. Rev. D 89 (2014) 035011 [arXiv: 1312.0582] [InSPIRE].

[47] C. Boehm, P.S.B. Dev, A. Mazumdar and E. Pukartas, Naturalness of light neutralino dark matter in pMSSM after LHC, XENON100 and Planck Data, JHEP 06 (2013) 113 [arXiv:1303.5386] [INSPIRE].

[48] N.E. Bomark, A. Kvellestad, S. Lola, P. Osland and A.R. Raklev, Long lived charginos in Natural SUSY?, JHEP 05 (2014) 007 [arXiv: 1310.2788] [INSPIRE].

[49] J. Fan and M. Reece, A new look at Higgs constraints on stops, JHEP 06 (2014) 031 [arXiv: 1401.7671] [INSPIRE].

[50] T. Gherghetta, B. von Harling, A.D. Medina and M.A. Schmidt, The price of being SM-like in SUSY, JHEP 04 (2014) 180 [arXiv:1401.8291] [INSPIRE].

[51] A. Delgado, M. Quirós and C. Wagner, General focus point in the MSSM, JHEP 04 (2014) 093 [arXiv: 1402.1735] [INSPIRE].

[52] A. Delgado, M. Quirós and C. Wagner, Focus point in the light stop scenario, Phys. Rev. D 90 (2014) 035011 [arXiv: 1406.2027] [InSPIRE].

[53] A. Fowlie, CMSSM, naturalness and the "fine-tuning price" of the very Large Hadron Collider, Phys. Rev. D 90 (2014) 015010 [arXiv:1403.3407] [InSPIRE]. 
[54] A. Mustafayev and X. Tata, Supersymmetry, naturalness and light higgsinos, Indian J. Phys. 88 (2014) 991 [arXiv: 1404.1386] [InSPIRE].

[55] I. Antoniadis, E.M. Babalic and D.M. Ghilencea, Naturalness in low-scale SUSY models and "non-linear" MSSM, Eur. Phys. J. C 74 (2014) 3050 [arXiv:1405.4314] [INSPIRE].

[56] J.A. Casas, J.M. Moreno, S. Robles, K. Rolbiecki and B. Zaldivar, What is a natural SUSY scenario?, arXiv:1407.6966 [INSPIRE].

[57] H. Baer, V. Barger, D. Mickelson, A. Mustafayev and X. Tata, Physics at a higgsino factory, JHEP 06 (2014) 172 [arXiv:1404.7510] [INSPIRE].

[58] CMS collaboration, Search for gluino mediated bottom- and top-squark production in multijet final states in pp collisions at 8 TeV, Phys. Lett. B 725 (2013) 243 [arXiv:1305.2390] [INSPIRE].

[59] CMS collaboration, Search for supersymmetry in hadronic final states with missing transverse energy using the variables $\alpha_{T}$ and b-quark multiplicity in pp collisions at $\sqrt{s}=8$ TeV, Eur. Phys. J. C 73 (2013) 2568 [arXiv:1303.2985] [INSPIRE].

[60] ATLAS collaboration, Search for supersymmetry at $\sqrt{s}=8$ TeV in final states with jets and two same-sign leptons or three leptons with the ATLAS detector, JHEP 06 (2014) 035 [arXiv:1404.2500] [INSPIRE].

[61] ATLAS collaboration, Search for squarks and gluinos with the ATLAS detector in final states with jets and missing transverse momentum using $\sqrt{s}=8$ TeV proton-proton collision data, JHEP 09 (2014) 176 [arXiv:1405.7875] [INSPIRE].

[62] ATLAS collaboration, Search for top squark pair production in final states with one isolated lepton, jets and missing transverse momentum in $\sqrt{s}=8 \mathrm{TeV}$ pp collisions with the ATLAS detector, JHEP 11 (2014) 118 [arXiv: 1407.0583] [INSPIRE].

[63] J.L. Feng, K.T. Matchev and D. Sanford, Focus point supersymmetry redux, Phys. Rev. D 85 (2012) 075007 [arXiv: 1112.3021] [INSPIRE].

[64] H. Baer, V. Barger and D. Mickelson, How conventional measures overestimate electroweak fine-tuning in supersymmetric theory, Phys. Rev. D 88 (2013) 095013 [arXiv: 1309. 2984] [INSPIRE].

[65] WMAP collaboration, G. Hinshaw et al., Nine-year Wilkinson Microwave Anisotropy Probe (WMAP) observations: cosmological parameter results, Astrophys. J. Suppl. 208 (2013) 19 [arXiv: 1212.5226] [INSPIRE].

[66] Planck collaboration, P.A.R. Ade et al., Planck 2013 results. XVI. Cosmological parameters, Astron. Astrophys. 571 (2014) A16 [arXiv:1303.5076] [INSPIRE].

[67] J.L. Feng, K.T. Matchev and F. Wilczek, Prospects for indirect detection of neutralino dark matter, Phys. Rev. D 63 (2001) 045024 [astro-ph/0008115] [INSPIRE].

[68] H. Baer, C. Balázs and A. Belyaev, Neutralino relic density in minimal supergravity with coannihilations, JHEP 03 (2002) 042 [hep-ph/0202076] [INSPIRE].

[69] A. Crivellin et al., Light stops, blind spots, and isospin violation in the MSSM, arXiv: 1503.03478 [INSPIRE].

[70] H. Baer, A. Belyaev, T. Krupovnickas and J. O'Farrill, Indirect, direct and collider detection of neutralino dark matter, JCAP 08 (2004) 005 [hep-ph/0405210] [INSPIRE]. 
[71] D.S.M. Alves, E. Izaguirre and J.G. Wacker, Where the sidewalk ends: jets and missing energy search strategies for the $7 \mathrm{TeV}$ LHC, JHEP 10 (2011) 012 [arXiv:1102.5338] [INSPIRE].

[72] C. Han et al., Probing light higgsinos in natural SUSY from monojet signals at the LHC, JHEP 02 (2014) 049 [arXiv:1310.4274] [INSPIRE].

[73] P. Schwaller and J. Zurita, Compressed electroweakino spectra at the LHC, JHEP 03 (2014) 060 [arXiv: 1312.7350] [INSPIRE].

[74] Z. Han, G.D. Kribs, A. Martin and A. Menon, Hunting quasidegenerate higgsinos, Phys. Rev. D 89 (2014) 075007 [arXiv:1401.1235] [InSPIRE].

[75] H. Baer, A. Mustafayev and X. Tata, Monojets and mono-photons from light higgsino pair production at LHC14, Phys. Rev. D 89 (2014) 055007 [arXiv:1401.1162] [INSPIRE].

[76] H. Baer, A. Mustafayev and X. Tata, Monojet plus soft dilepton signal from light higgsino pair production at LHC14, Phys. Rev. D 90 (2014) 115007 [arXiv:1409.7058] [INSPIRE].

[77] CMS collaboration, Search for dark matter, extra dimensions and unparticles in monojet events in proton-proton collisions at $\sqrt{s}=8 \mathrm{TeV}$, Eur. Phys. J. C 75 (2015) 235 [arXiv: 1408.3583] [INSPIRE].

[78] ATLAS collaboration, Search for new phenomena in monojet plus missing transverse momentum final states using $10 \mathrm{fb}^{-1}$ of pp collisions at $\sqrt{s}=8 \mathrm{TeV}$ with the ATLAS detector at the LHC, ATLAS-CONF-2012-147 (2012).

[79] ATLAS collaboration, Sensitivity to WIMP dark matter in the final states containing jets and missing transverse momentum with the ATLAS detector at $14 \mathrm{TeV}$ LHC, ATL-PHYS-PUB-2014-007 (2014).

[80] A. Belyaev, The interplay of the LHC and direct dark matter detection in unravelling natural supersymmetry at the focus point, presentation at the $22^{\text {nd }}$ International Conference on Supersymmetry and Unification of Fundamental Interactions (SUSY2014), July 21-26, Manchester, U.K. (2014).

[81] C. Han, D. Kim, S. Munir and M. Park, Accessing the core of naturalness, nearly degenerate higgsinos, at the LHC, JHEP 04 (2015) 132 [arXiv:1502.03734] [INSPIRE].

[82] N. Arkani-Hamed, A. Delgado and G.F. Giudice, The well-tempered neutralino, Nucl. Phys. B 741 (2006) 108 [hep-ph/0601041] [INSPIRE].

[83] A. Bartl, W. Majerotto and W. Porod, Large Higgs boson exchange contribution in three-body neutralino decays, Phys. Lett. B 465 (1999) 187 [hep-ph/9907377] [INSPIRE].

[84] A. Djouadi and Y. Mambrini, Three body decays of SUSY particles, Phys. Lett. B 493 (2000) 120 [hep-ph/0007174] [INSPIRE].

[85] A. De Simone, V. Sanz and H.P. Sato, Pseudo-Dirac dark matter leaves a trace, Phys. Rev. Lett. 105 (2010) 121802 [arXiv:1004.1567] [INSPIRE].

[86] A.E. Nelson, N. Rius, V. Sanz and M. Ünsal, The minimal supersymmetric model without a $\mu$ term, JHEP 08 (2002) 039 [hep-ph/0206102] [INSPIRE].

[87] K. Hsieh, Pseudo-Dirac bino dark matter, Phys. Rev. D 77 (2008) 015004 [arXiv: 0708.3970] [INSPIRE].

[88] G. Bélanger, K. Benakli, M. Goodsell, C. Moura and A. Pukhov, Dark matter with dirac and Majorana gaugino masses, JCAP 08 (2009) 027 [arXiv: 0905.1043] [INSPIRE]. 
[89] ATLAS collaboration, Search for charginos nearly mass degenerate with the lightest neutralino based on a disappearing-track signature in pp collisions at $\sqrt{s}=8 \mathrm{TeV}$ with the ATLAS detector, Phys. Rev. D 88 (2013) 112006 [arXiv:1310.3675] [INSPIRE].

[90] CMS collaboration, Search for disappearing tracks in proton-proton collisions at $\sqrt{s}=8$ TeV, JHEP 01 (2015) 096 [arXiv: 1411.6006] [INSPIRE].

[91] A. De Simone, J. Fan, V. Sanz and W. Skiba, Leptogenic supersymmetry, Phys. Rev. D 80 (2009) 035010 [arXiv:0903.5305] [INSPIRE].

[92] ATLAS collaboration, Searches for heavy long-lived charged particles with the ATLAS detector in proton-proton collisions at $\sqrt{s}=8 \mathrm{TeV}$, JHEP 01 (2015) 068 [arXiv: 1411.6795] [INSPIRE].

[93] Planck collaboration, P. Ade et al., Planck 2015 results. XIII. Cosmological parameters, arXiv: 1502.0158.

[94] R. Allahverdi, B. Dutta and K. Sinha, Non-thermal higgsino dark matter: cosmological motivations and implications for a 125 GeV Higgs, Phys. Rev. D 86 (2012) 095016 [arXiv:1208.0115] [INSPIRE].

[95] G. Bélanger, F. Boudjema, A. Pukhov and A. Semenov, MicrOMEGAs 2.0: a program to calculate the relic density of dark matter in a generic model, Comput. Phys. Commun. 176 (2007) 367 [hep-ph/0607059] [INSPIRE].

[96] G. Bélanger et al., Indirect search for dark matter with MicrOMEGAs2.4, Comput. Phys. Commun. 182 (2011) 842 [arXiv: 1004.1092] [INSPIRE].

[97] LUX collaboration, D.S. Akerib et al., First results from the LUX dark matter experiment at the Sanford Underground Research Facility, Phys. Rev. Lett. 112 (2014) 091303 [arXiv: 1310.8214] [INSPIRE].

[98] XENON1T collaboration, E. Aprile, The XENON1T dark matter search experiment, Springer Proc. Phys. C12-02-22 (2013) 93 [arXiv: 1206.6288] [INSPIRE].

[99] Fermi-LAT collaboration, M. Ackermann et al., Constraining dark matter models from a combined analysis of Milky Way satellites with the Fermi Large Area Telescope, Phys. Rev. Lett. 107 (2011) 241302 [arXiv:1108.3546] [INSPIRE].

[100] S. Arrenberg et al., Working group report: dark matter complementarity, arXiv:1310.8621 [INSPIRE].

[101] CTA Consontium collaboration, M. Actis et al., Design concepts for the Cherenkov Telescope Array CTA: an advanced facility for ground-based high-energy gamma-ray astronomy, Exper. Astron. 32 (2011) 193 [arXiv: 1008.3703] [INSPIRE].

[102] CTA collaboration, M. Doro et al., Dark matter and fundamental physics with the Cherenkov Telescope Array, Astropart. Phys. 43 (2013) 189 [arXiv:1208.5356] [INSPIRE].

[103] M. Cahill-Rowley et al., Complementarity of dark matter searches in the phenomenological MSSM, Phys. Rev. D 91 (2015) 055011 [arXiv:1405.6716] [INSPIRE].

[104] CMS Collaboration, Search for new physics in monojet events in pp collisions at $\sqrt{s}=8 \mathrm{TeV}$, CMS-PAS-EXO-12-048 (2012).

[105] J. Alwall, M. Herquet, F. Maltoni, O. Mattelaer and T. Stelzer, MadGraph 5: going beyond, JHEP 06 (2011) 128 [arXiv:1106.0522] [INSPIRE]. 
[106] N.D. Christensen and C. Duhr, FeynRules - Feynman rules made easy, Comput. Phys. Commun. 180 (2009) 1614 [arXiv:0806.4194] [InSPIRE].

[107] C. Degrande et al., UFO - The Universal FeynRules Output, Comput. Phys. Commun. 183 (2012) 1201 [arXiv: 1108.2040] [INSPIRE].

[108] A. Belyaev, N.D. Christensen and A. Pukhov, CalcHEP 3.4 for collider physics within and beyond the standard model, Comput. Phys. Commun. 184 (2013) 1729 [arXiv:1207.6082] [INSPIRE].

[109] J. Pumplin et al., New generation of parton distributions with uncertainties from global QCD analysis, JHEP 07 (2002) 012 [hep-ph/0201195] [INSPIRE].

[110] T. Sjöstrand, S. Mrenna and P.Z. Skands, PYTHIA 6.4 physics and manual, JHEP 05 (2006) 026 [hep-ph/0603175] [INSPIRE].

[111] DELPHES 3 collaboration, J. de Favereau et al., DELPHES 3, a modular framework for fast simulation of a generic collider experiment, JHEP 02 (2014) 057 [arXiv:1307.6346] [INSPIRE].

[112] G. Brooijmans et al., Les Houches 2013: physics at TeV colliders: new physics working group report, arXiv: 1405.1617 [INSPIRE].

[113] ATLAS collaboration, G. Aad et al., Search for new phenomena in final states with an energetic jet and large missing transverse momentum in pp collisions at $\sqrt{s}=8 \mathrm{TeV}$ with the ATLAS detector, arXiv:1502.0151.

[114] S. Bityukov and N. Krasnikov, On observability of signal over background, physics/9809037 [IFVE-98-48].

[115] O. Buchmueller, M.J. Dolan and C. McCabe, Beyond effective field theory for dark matter searches at the LHC, JHEP 01 (2014) 025 [arXiv: 1308.6799] [INSPIRE].

[116] G. Busoni, A. De Simone, E. Morgante and A. Riotto, On the validity of the effective field theory for dark matter searches at the LHC, Phys. Lett. B 728 (2014) 412

[arXiv: 1307.2253] [INSPIRE]. 